\title{
Metagenomic Analysis of Rhizosphere Microbiome Provides Insights Into Occurrence of Iron Deficiency Chlorosis in Field of Asian Pears
}

Bing Jia

Anhui Agricultural University

Xiao Chang

Anhui Agricultural University

Yuanyuan Fu

Anhui Agricultural University

Wei Heng

Anhui Agricultural University

Zhenfeng Ye

Anhui Agricultural University

Pu Liu

Anhui Agricultural University

Li Liu

Anhui Agricultural University

Yosef Al Shoffe

Cornell University

Christopher Watkins

Cornell University

Liwu Zhu ( $\sim$ zhuliwu@ahau.edu.cn )

Anhui Agricultural University

\section{Research Article}

Keywords: Pear, Fe-deficiency chlorosis, Rhizosphere soil, Microbiome, Metagenomic analysis

Posted Date: December 9th, 2020

DOI: https://doi.org/10.21203/rs.3.rs-116420/v1

License: (1) (1) This work is licensed under a Creative Commons Attribution 4.0 International License.

Read Full License 
Version of Record: A version of this preprint was published at BMC Microbiology on January 8th, 2022. See the published version at https://doi.org/10.1186/s12866-021-02432-7. 


\section{Abstract}

Fe-deficiency chlorosis (FDC) of Asian pear plants is widespread, but little is known about the association between the bacterial biogeography in the rhizosphere soil and leaf chlorosis. The leaf mineral concentration, leaf subcellular structure, soil physiochemical properties, and bacterial species community and distribution have been analyzed. The total Fe in leaves with Fe-deficiency was positively correlated with total $\mathrm{K}, \mathrm{Mg}, \mathrm{S}, \mathrm{Cu}, \mathrm{Zn}, \mathrm{Mo}$ and $\mathrm{Cl}$ contents, but no differences of available Fe (AFe) were detected between the rhizosphere soil of chlorotic and normal plants. Degraded ribosomes and degraded thylakloid stacks in chloroplast were observed in chlorotic leaves. Bacterial community and distribution patterns in the rhizosphere soil of chlorotic plants were distinct from those of normal plants and the relative abundance and microbiome diversity were more stable in the rhizosphere soils of normal than in chlorotic plants. Water-impermeable tables reduce the soil aeration, inhibit root growth, and cause some absorption root death from infection by Fusarium solani. The rhizosphere soils of FDC plants have distinct composition, lower relative abundance, and unstable diversity of microbiome. Higher amounts of AFe in the rhizosphere soil of chlorotic plants demonstrated it was the Fe uptake that caused FDC in this study.

\section{Introduction}

China is the largest Asian pear fruit producing country in world with annual production of $16,200 \mathrm{k}$ tones, accounting for $68.2 \%$ of the world production ${ }^{1}$. Over the last three decades, poor agricultural practices, such as unsustainable fertilization and herbicide application, have resulted in the increasing occurrence of Fe-deficiency chlorosis (FDC) and associated economic losses. Fe is essential both for productivity and quality of plants because it is association with photosynthetic efficiency ${ }^{2}$. Fe-deficiency is one of the most common nutrient constraints that is associated with development of chlorosis of plant leaves growing in calcareous and/or alkaline soils ${ }^{3-6}$, although manganese, zinc, or calcium deficiencies in the plant will also cause chlorosis ${ }^{7,8}$. Fe is also necessary for many enzyme functions involved with plant metabolism ${ }^{9,10}$. Dicots respond to Fe-deficiency by releasing protons into the soil environment and increasing the reducing capacity of the rhizodermal cells ${ }^{11}$. Fe can only be absorbed as an available ferrous iron $\left(\mathrm{Fe}^{2+}\right)$, a soil $\mathrm{pH}$ in the range of 7.5-8.5 resulting in low solubility and slow dissolution kinetics of Fe-bearing minerals ${ }^{12,13}$.

Microbial community assembly in the rhizosphere is governed by abiotic and biotic factors, such as soil type, climate change, anthropogenic activities, plant host genotype/cultivar, and pathogenic and mutualistic-symbiotic organisms ${ }^{14}$. In natural ecosystems, the plant species is likely to be a more important determinant of the rhizosphere microbial community than soil type $\mathrm{e}^{15}$. In contrast, the importance of the rhizosphere microbiota for plant growth and health in agricultural ecosystems is lower compared with that in natural ecosystems owing to the input of fertilizers and pesticides ${ }^{16}$. Microbes have developed various adaptations to thrive in the rhizospheric soil and the host genotype accounts for approximately $5.7 \%$ of the variance in the rhizosphere microbiome composition ${ }^{17,18}$. 
Progress has been made in the role of soil microorganisms in plant mineral nutrition ${ }^{19}$ and the knowledge on composition of rhizospheric microbiota and their dynamics ${ }^{20}$. The plant microbiome contains pathogens, beneficial and neutral microbes, all of which contribute to host fitness and adaptability to the environment ${ }^{21}$. Plant hosts and their developmental stages play an important role in shaping the structure of rhizosphere microbiome ${ }^{22,23}$, most probably by root exudates ${ }^{24}$. Drought also shifts the composition of root microbial communities in many grass species ${ }^{25,26}$. Host genetic influences on the richness of the microbiomes in rhizosphere soil were shown for maize $e^{27-29}$. The rhizosphere microbiome of cotton varied with soil type, genotype and developmental stage ${ }^{30}$. However, whether the bacterial composition in rhizosphere soil is altered to cope with the incidence of FDC in Asian pears is unknown.

The objective of this work was to investigate the differences of subcellular structure and Fe concentration level Asian pear leaves with and without Fe-deficiency chlorosis, and ascertain if the alteration of microbiome diversity in the rhizosphere soil is related to the incidence of Fe-deficiency chlorosis in the plant.

\section{Results}

\section{Mineral element content and subcellular structure of leaves}

Compared with the normal leaves, the total ferric iron (TFe) concentrations in leaves with severe, moderate, and mild Fe-deficiency were reduced by $77 \%, 62 \%$, and $59 \%$, respectively (Table S1).

Correlation analysis of mineral contents showed that the content of TFe in leaves with Fe-deficiency was positively correlated with the contents of total $\mathrm{K}, \mathrm{Mg}, \mathrm{S}, \mathrm{Cu}, \mathrm{Zn}, \mathrm{Mo}$ and $\mathrm{Cl}$, but negatively correlated with Ca content (Table S2). Therefore, the different degrees of Fe-deficiency chlorosis varied greatly with not only the Fe content itself but also with most of other elements in the leaves.

TEM revealed that chlorotic leaves had smaller chloroplasts and larger vacuoles compared with the subcellular structure of normal leaves. Also, degraded ribosomes, degraded stacks of thylakloids in the smaller chloroplast, and normal mitochondria were observed on the transverse section of chlorotic leaves (Fig. 1a, b and c). In contrast, normal ribosomes, stacks of thylakloids in chloroplast, and mitochondria, were found in normal leaves (Fig. 1d, e and f).

\section{Physicochemical properties of the rhizosphere soil}

No significant differences of $\mathrm{OM}, \mathrm{pH}$ values, and $\mathrm{CEC}$ in the rhizosphere soil between chlorotic and normal plants were detected (Fig. 2a). However, NN, AK, ECa, and EMg in the rhizosphere soil of chlorotic plants were higher than that of normal plants, but there was no significant differences of the TN, AhN, AN, AP, and TS contents in the rhizosphere soil between the two plant types (Fig. 2b and c).

$\mathrm{ACu}$ and $\mathrm{AMn}$ in the rhizosphere soil of chlorotic plants were lower than that of normal plants. It was interesting that the contents of TFe and AFe in the rhizosphere soil of chlorotic plants were higher than in 
that of normal plants, although there was no significant difference of the AFe content in the rhizosphere soil between the two plant types (Fig. 2d).

\section{Number of genes and GO function and pathway enrichment in the rhizosphere soil}

After the metagenomic sequencing of the six samples (CH1-3 and CK1-3), an average of $7.12 \mathrm{Gbp}$ clean data was generated per sample, and a total of 166,658 non-redundant genes were identified. The average of total and annotated genes in the rhizosphere soil of chlorotic plant was 84,605 and 64,317 , respectively, which was 18,779 and 13,182 genes more than that of normal plants, respectively (Supplementary Table S3).

Quantitative and differential analysis of gene abundance in CK compared with $\mathrm{CH}$ identified 5,552 total DEGs; of these 4,624 genes were high-abundance and 928 genes were low-abundance in the rhizosphere soil of chlorotic plants. The DEGs between the rhizosphere soil of chlorotic and normal plants were categorized into $37 \mathrm{GOs}$. Among the biological process categories, those associated with metabolic processes was the largest class, including 1,422 genes and accounting for $25.6 \%$ of the total DEGs), followed by cellular processes $(1,214 ; 21.9 \%)$, single-organism processs $(999 ; 18.0 \%)$, localization $(224$; $4.0 \%)$, biological regulation $(207 ; 3.7 \%)$, and regulation of biological processes $(203 ; 3.7 \%)$. In the molecular function category, 1,961 (35.3\%) and 982 (17.7\%) DEGs were classified into catalytic activity and binding, respectively, followed by transporter activity $(133 ; 2.4 \%)$. With respect to the cellular component category, membrane were the largest class $(580 ; 10.4 \%)$, followed by cell $(463 ; 8.4 \%)$, cell part (456; 8.2\%), membrane parts $(261 ; 4.7 \%)$, and macromolecular complex (87; $1.6 \%)$ (Fig. 3$)$.

After the DEGs were subjected to KEGG enrichment analysis, there were 23 pathways enriched in CK vs $\mathrm{CH}$. Compared with that of normal plants, the main enriched DEGs in the rhizosphere soil of chlorotic plants were involved in metabolic pathways (1,090 DEGs; $33.9 \%)$, biosynthesis of secondary metabolites (495 DEGs; 15.4\%), purine metabolism (159 DEGs; 5.0\%), alanine, aspartate and glutamate metabolism (77 DEGs; 2.4\%), homologous recombination (47 DEGs; 1.5\%), and mismatch repair pathways (42 DEGs; 1.3\%) (Supplementary Table S4; Fig. 4).

\section{Microbiota in the rhizosphere soil}

The community compositions of annotated microbiome indicated that there were 5 kingdoms, 52 phyla, 94 classes, 206 orders, 404 families, 1,161 genera, and 3,043 species in the rhizosphere soil of chlorotic plants; it was one phylum less and one order, 11 families, 59 genera, and 313 species more than in that of normal plant (Supplementary Table S3).

Fifteen phyla were observed at relatively high abundance, which was defined as having a relative abundance greater than $0.5 \%$ in any one sample from chlorotic and normal plants, beside the unclassified (accounting for $30.6 \%$ and $29.9 \%$ of the total in chlorotic and normal plants, respectively) and the others $(1.4 \% ; 1.5 \%)$. Notably, the top five phyla dominated in the rhizophere soil of chlorotic plants accounted for $62.1 \%$ of the total: Proteobacteria (42.7\%), Thaumarchaeota (12.4\%), Acidobacteria 
(3.2\%), Actinobacteria (2.1\%), and Firmicutes (1.8\%); while that of normal plant accounted for $60.6 \%$ of the total: Proteobacteria (47.5\%), Thaumarchaeota (4.4\%), Gemmatimonadetes (3.8\%), Nitrospirae (2.5\%) and Actinobacteria (2.4\%). Among these top five phyla, three of them were identical and two of them were different (Fig. 5a; Table S5).At the level of genus, Nitrososphaera (2.5\%), Nitrospira (2.4\%), Gemmatirosa (2.2\%), Pseudomonas (1.0\%), and Burkholderia ( $0.8 \%$ ) were the top five genera from sixteen ones with an average relative abundance greater than $0.5 \%$ in any one sample of the rhizosphere soil from normal plant; while that from chlorotic plants Candidatus Nitrosoarchaeum (4.0\%), Nitrosopumilus (1.6\%), Pseudomonas (0.9\%), Gemmatirosa (0.8\%), and Nitrospira (0.7\%) were the top five genera, two of them were different and three of them were identical but at different relative abundance (Fig. 5b).

Compared with the rhizosphere soil of normal plant, the relative abundance of the top 12 species in the rhizosphere soil of chlorotic plants showed that Candidatus Nitrosoarchaeum koreensis was the most dominant species with the average relative abundance of $2.40 \%$ and an increase of $80.0 \%$, followed by Gemmatirosa kalamazoonesis $(0.85 \%$; and $60.8 \%)$, Candidatus Nitrosoarchaeum limnia $(0.84 \%$; and 78.0\%), Nitrospira defluvii (0.74\%; and 69.0\%), Candidatus Solibacter usitatus (0.67\%; and $44.5 \%)$, Candidatus Nitrosopumilus salaria (0.58\%; and $84.5 \%)$, Chloracidobacterium thermophilum $(0.51 \%$; and

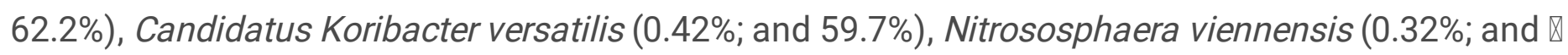
$87.1 \%)$, Sulfuricella denitrificans ( $0.29 \%$; and $34.7 \%)$, uncultured Acidobacteria bacterium $(0.26 \%$; and $53.9 \%)$, and Gemmatimonas aurantiaca $(0.20 \%$; and $61.43 \%)$, beside the uncultured bacterium $(2.47 \%$; and $28.0 \%$ ), the others (19.30\%; and $4.2 \%$ ), and the unclassified species $(70.16 \%$; and $2.3 \%)$. For in CK vs $\mathrm{CH}$ the relative abundance was different only for $C$. N. koreensis, G. kalamazoonesis, C. N. limnia, G. aurantiaca, and C. N. salaria (Fig. 5c; Table S6).

\section{Characterization of bacterial communities and simplified species compositions}

The rhizosphere soil of normal plants had greater diversity of the bacterial community than that of chlorotic plants (Fig. 6). Species diversity analysis showed that the bacterial community in the rhizosphere soil of normal plant had a higher and less variable Shannon index (Mean \pm SE $=2.403 \pm$ $0.006)$ than in that of chlorotic plant (Mean $\pm S E=2.330 \pm 0.142$ ).

PCA revealed that a relatively large portion of the total variance contributed to the first two PCs based on expressed genes annotated $(\mathrm{PC}-1=41.32 \%$ and $\mathrm{PC}-2=23.22 \%$ ) and abundance of taxonomic species (PC-1 $=43.65 \%$ and PC-2 $=34.48 \%$ ), respectively. The two distinctive groups (CK1-3, normal plants; $\mathrm{CH} 1-3$, chlorotic plants) was formed, not only by the number of expressed genes annotated (Fig. 7a), but also by the abundance of taxonomic species (Fig. 7b).

By synthetic analysis of the species presented in all samples with relative abundance higher than $0.6 \%$, we obtained the most simplified communities. In the rhizosphere soil of chlorotic plants the top five species were Nitrospira defluvii, Gemmatirosa kalamazoonesis, Sulfuricella denitrificans, Candidatus Nitrosoarchaeum koreensis, and Candidatus Koribacter versatilis), while in that of normal plants the top five species were N. defluvii, G. kalamazoonesis, S. denitrificans, G. aurantiaca, and C. S. usitatus. N. defluvii, G. kalamazoonesis, and S. denitrificans were the same in both rhizosphere soils, but their relative 
abundance decreased by $48 \%, 37 \%$, and $22 \%$, respectively, and two of them (G. aurantiaca and $C$. S. usitatus) were substituted by an ammonia-oxidizing soil archaeon, $C$. N. koreensis and a nitrite and nitrate reduction related species, C. K. versatilis in that of chlorotic plants (Fig. 8).

\section{Water-impermeable table formed by silt stratification inhibit root growth}

A water-impermeable table formed by silt stratification was found in the chlorotic plants but not the normal plants. As for root growth, it is normal in the upper layer of soil and some absorption roots in the middle and lower layers of soil were decayed (Supplementary Fig. 2).

A total of 5 fungi species were isolated from the roots of chlorotic plants; four of them were pathogenic fungus and one of them was non-pathogenic. In contrast, no pathogenic fungi were isolated and only one non-pathogenic is identified from the roots of normal plant. No pathogenic bacterium was identified in normal plants, while a single non-pathogenic bacterium species was found in the roots of chlorotic plants (Nigrospora oryzae) and normal plants (Neurospora intermedia) (Table 1).

Table 1 Fungal and bacterial species identified in the roots of chlorotic and normal plants

\begin{tabular}{|lll|}
\hline Microorganism & Roots of Chlorotic Plant & Roots of Normal Plant \\
\hline Non-pathogenic Fungus & Nigrospora oryzae & Neurospora intermedia \\
\hline Pathogenic Fungus & Fusarium solani & - \\
\hline & Fusarium phaseoli & - \\
\hline Pythium spinosum & - \\
\hline Non-pathogenic Bacterium & Paenibacillus lautus & Bacillus flexus \\
\hline & Bacillus niacini & Bacillus pumilus \\
\hline Bacillus gibsonii & Paenibacillus sp. \\
\hline- & Lysinibacillus fusiformis \\
\hline
\end{tabular}

\section{Discussion}

Fe-deficiency enlarged the epidermal cells and reduced the guard cell size, bundle sheaths appeared disorganized, and the mesophyll was more compacted and less porous compared to normal (healthy) pear leaves ${ }^{31}$. However, there have not been previous reports of ribosomal degradation before the stacks of thylakloids began to destruct in chloroplast observed on the ultrathin transverse section of leaves with FDC. 
Inorganic cation and anion concentrations in apoplastic fluid of sugar beet leaves increased with $\mathrm{Fe}$ deficiency, and the largest increase in apoplastic fluid concentrations with Fe deficiency was 20-fold for $\mathrm{Ca}$, followed by 3.5-fold for $\mathrm{Mg}$, and 1.7-fold for $\mathrm{K}^{32}$. Our results show that 'Whangkeumbea' pear leaves with Fe-deficiency were negatively correlated only with Ca content, but not those of Mg and K. In Arabidopsis seeds, only $5 \%$ of $\mathrm{Fe}$ is ferritin-bound and its main storage compartment is the vacuole ${ }^{33}$; up to $80 \%$ of the cellular Fe was found in the chloroplast, which is consistent with its major function in photosynthesis ${ }^{34}$. But the mechanism by which chloroplasts become smaller while the vacuole increases in size of leaves with FDC remains to be clarified.

In pear plants, Fe-deficiency chlorosis was associated with decreased leaf chlorophyll concentrations, fruit number, and total fruit weight per tree by 36,72 , and $64 \%$, respectively ${ }^{35,36}$. Fe-deficiency may occur because there is an insufficient Fe amount in the soil[8,34]. Fe is unavailable because of high $\mathrm{pH}$ in alkaline soils ${ }^{7}$, or the Fe may not be absorbed due to the root damage by soilborne diseases ${ }^{37}$. However, in our study, TFe and AFe contents in the rhizosphere soil of chlorotic plants were higher than those of normal plants, and there were no significant differences of $\mathrm{pH}$ values in the rhizosphere soil between chlorotic and normal plants. It was demonstrated that Fe concentration, and high $\mathrm{pH}$ value in the soil were not the possible causes of Fe-deficiency chlorosis in this study.

Nitrification is a two-step process where ammonia is considered to first be oxidized to nitrite by ammoniaoxidizing bacteria and/or archaea, and then to nitrate by nitrite-oxidizing bacteria. Nitrification can be completed by a Nitrospira bacterium Ca. N. nitrosa, Ca. N. nitrificans ${ }^{38}$, and Ca. N. inopinata ${ }^{39}$. Their genomes contain the full set of ammonia monooxygenase and hydroxylamine dehydrogenase genes for ammonia oxidation, in addition to the nitrite oxidoreductase subunits necessary for nitrite oxidation in Nitrospira. However, none of these species was found in the rhizosphere soil of the plant in our study.

Plants release one proton $\left(\mathrm{H}^{+}\right)$for every ammonium $\left(\mathrm{NH}_{4}^{+}\right)$taken up resulting in a reduction $\mathrm{pH}$ in rhizosphere. When supplied with nitrate $\left(\mathrm{NO}_{3}{ }^{\square}\right)$, the plant releases bicarbonate, which will increase the rhizosphere $\mathrm{pH}$. These changes in $\mathrm{pH}$ can influence Fe availability in the soil ${ }^{11}$. The $\mathrm{NO}_{3}{ }^{\square}$ content in the rhizosphere soil of chlorotic plants was higher but the $\mathrm{pH}$ value was lower than that of normal plant. It is possible that the amount of $\mathrm{NO}_{3}{ }^{\square}$ supply was insufficient to increase the $\mathrm{pH}$ in rhizosphere soil of chlorotic plants.

Among the top five bacterial species, three of them ( $N$. defluvii, G. kalamazoonesis, and S. denitrificans) were identical in in the rhizosphere soils of chlorotic and normal plants, but with lower relative abundance Two of them ( $G$. aurantiaca and $C$. S. usitatus) in soils of normal plants were substituted by $C . N$. koreensis and C. K. versatilis in soils of chlorotic plants.

defluvii is a nitrite-oxidizing bacterium firstly enriched from activated sludge from a municipal wastewater treatment plant ${ }^{40}$, which catalyzes aerobic nitrite oxidation and is adapted to live under significant substrate limitation with half-saturation constants $(K m) 9 \mu \mathrm{M} \mathrm{NO}_{2}{ }^{\square 41}$. The highest proportions of 
Gemmatimonadetes strains were isolated in arid soils, suggesting an adaption to low soil moisture ${ }^{42}$; it is found in a wide variety of environments and is particularly abundant in soils ${ }^{43}$. G. kalamazoonesis is a microaerophillic or aerobic bacterium belonging to the phylum Gemmatimonadetes. It does not produce catalase, and was isolated initially from agricultural soil in Kalamazoo, Michigan, United States ${ }^{44} . S$. denitrificans is a sulfur-oxidizing bacterium isolated from anoxic water of a freshwater lake in Japan ${ }^{45}$, which is microaerobic and could also utilize $\mathrm{NO}_{3}{ }^{\square}$ as an electron acceptor, reducing it to nitrogen ${ }^{46}$.

aurantiaca was present in all samples of rhizosphere soil of normal plant but was absent in the soil of chlorotic plants. It is an aerobic bacterium isolated first from an anaerobic-aerobic sequential batch reactor operated under enhanced biological phosphorus removal conditions for wastewater treatmen ${ }^{47}$.

Aerobic bacteria require oxygen to live and grow, while microaerophillic and microaerobic bacteria require very little oxygen to maintain growth. The greatly reduced abundance or absence of the three bacteria species $N$. defluvii, G. kalamazoonesis, and $S$. denitrificans from the rhizosphere soils of chlorotic plants may result from adverse soil aeration. Waterlogging or/and poor drainage of the soil may inhibit Fe uptake. After the field investigations were completed, we found water-impermeable tables formed by silt stratification in middle layer of soil at the chlorotic plants. This would reduce the aeration of middle and lower layers of soil, and result in poor root growth caused mainly by the dominant pathogenic species $F$. solani ${ }^{37}$, leading to the Fe-deficiency chlorosis of pear plants in this study.

C. S. usitatus is a $\mathrm{NO}_{2}$ and $\mathrm{NO}_{3}$ reduction species isolated from soil in ryegrass grass/clover pasture in Australia $^{48}$, and its genome contains not only nirA encoding nitrite reductase but also genes associated this uptake of $\mathrm{Fe}$, including the genes $f e o A B$, which encode a high-affinity ferrous $\left(\mathrm{Fe}^{2+}\right)$ iron transporter ${ }^{49}$. C. K. versatilis is also a $\mathrm{NO}_{2}$ and $\mathrm{NO}_{3}$ reduction species ${ }^{50}$, which contain genes encoding iron permease FTR1 and FTR1 family proteins as well as sequences similar to fet $3 p^{49}$, which encodes a multicopper oxidase involved in FTR1-mediated iron uptake in yeast ${ }^{51}$. Ca. K. versatilis and $\mathrm{Ca}$. S. usitatus, are present in the rhizosphere soil of chlorotic and normal plants, respectively; their genomes contain genes encoding candidate siderophore receptors and other Fe transporter genes suggesting that they may be involved in $\mathrm{Fe}^{2+}$ uptake.

C. N. koreensis is an ammonia-oxidizing soil archaea belonging to the phylum Thaumarchaeota, which is an anaerobic bacterium and ubiquitous microbes in marine, freshwater, and terrestrial environments ${ }^{52}$. However, anaerobic bacteria generate energy by the oxidation of $\mathrm{Fe}^{2+}$ compounds to $\mathrm{Fe}^{3+53}$. As an anaerobic bacterium, $\mathrm{Ca}$. N. koreensis is present mainly in the rhizosphere soil of chlorotic plants but it is absent from that of normal plants. This suggests that it may be involved decrease of Fe availability in rhizosphere soil for plant absorption, although the concentrations of TFe and AFe tested in this study were higher in the rhizosphere soil of chlorotic plants.

Some bacteria, such as Ca. K. versatilis and Ca. S. usitatus, can scavenge Fe via excreted siderophores. It still needs to be proven whether or not these bacteria have potential advantage to compete Fe with roots 
in rhizosphere soil, where $\mathrm{Fe}$ is bound to soil colloids and the available concentration is limited to pear plant growth.

\section{Materials And Methods}

\section{Leaf sample preparation}

Asian pear (Pyrus pyrifolia Nakai) plants with or without Fe-deficiency chlorosis were surveyed in successive three years (2016-2018) at the Old Yellow River Valley Orchard (116.92_E; 34.34_N), located in Xiaoxian county, Anhui province, China, where the annual precipitation is about $800 \mathrm{~mm}$. Forty year-old 'Whangkeumbea' pear trees grafted onto rootstock of $P$. betulaefolia Bge., grown in sandy loam soils, and with drip irrigation were used for these experiments. According to the degree of leaf chlorosis, 60 mature leaves with mild (lighter-green to lime-green color of interveinal tissue, Fig. S1A), moderate (yellow color of interveinal tissue, Fig. S1 B), and severe (yellow leaf veins, Fig. S1C) symptoms were taken from plants with chlorosis (Fig. S1D), respectively; control leaves (Fig. S1E) were taken from normal plants on June 20, 2018 (Fig. S1F), and then, brought back to the laboratory and divided into two groups, one for the leaf transversal sections, and one for cleaning, drying and grinding to determine the contents of $N, P, K, C a$, $\mathrm{Mg}$, and $\mathrm{S}$ in percentage on a dry weight (DW) basis, and $\mathrm{Fe}, \mathrm{B}, \mathrm{Mn}, \mathrm{Cu}, \mathrm{Zn}, \mathrm{Mo}$ and $\mathrm{Cl}$, expressed as $\mathrm{mg} \cdot \mathrm{kg}^{-1}$ on a dry weight basis at the Institute of Geology and Mineral Resources, Department of Land and Resources of Anhui Province, Hefei, China.

\section{Soil sample collection}

Rhizosphere soil was defined as that tightly bounded to the root surface ${ }^{16}$. Each composite soil sample was collected on August 20, 2018 at the rhizosphere sites from the east, south, west, and north sides, from three replicate trees with leaves exhibiting Fe-deficiency chlorosis ( $\mathrm{CH} 1-3)$ and normal plants as control (CK1-3). All the samples were frozen in liquid nitrogen, packed into sterile polyethylene bags and immediately transported on ice packs to the laboratory. The soils were sieved through $2 \mathrm{~mm}$ mesh to remove visible roots, residues and stones. Each replicate was then divided into two parts: one was stored at $4^{\circ} \mathrm{C}$ for the analyses of soil physiochemical properties while the other part was stored at $-80^{\circ} \mathrm{C}$ within a week for DNA extraction and metagenomic analysis of the rhizosphere microbiome.

\section{Leaf subcellular structure observation}

The leaf pieces were treated with $2.5 \%$ glutaraldehyde solution under vacuum until the leaf pieces sank to the bottom of the bottle, and fixed at $4^{\circ} \mathrm{C}$ for 24 hours. The pieces were rinsed with $0.1 \mathrm{M} \mathrm{pH} 7.0$ phosphate buffers 3 times for 15 min; fixed with $1 \%$ osmium tetroxide for $1-2 \mathrm{~h}$, rinsed 3 for 15 min with $0.1 \mathrm{M} \mathrm{pH} 7.0$ phosphate buffer. After dehydration in a graded ethanol series $(50,70,90$, and $100 \%)$ for 10 min at each step, the pieces were embeded in Epon 812 epoxy resin. Cross sections were then taken by a 
microtome (LKB-V, Uppsala, Sweden) at a thickness of 70-90 nm. Finally, the cross sections were stained with uranyl acetate-lead citrate and imaged with a transmission electron microscope (TEM) (Hitachi 7650 , Tokyo, Japan) operating at an accelerating voltage of $80 \mathrm{kV}$. Images were acquired at the magnifications of $1.0,4.0$, and $8.0 \mathrm{k}^{54}$.

\section{Analyses of soil physiochemical properties}

Soil $\mathrm{pH}$ was determined with a soil to water ratio of 1:5 using a pH monitor (Orion-868, Thermo-Fisher Scientific, city, USA). The soil was air dried and sieved (1 mm mesh). Organic matter (OM) was determined using the potassium dichromate-sulphuric acid heating method ${ }^{55}$, and cation exchange capacity (CEC) was determined by titration with standard sodium hydroxide solution ${ }^{56}$. The total nitrogen (TN) content was analyzed by the Kjeldahl method, while the alkaline-hydrolysable nitrogen (AhN) was measured by the hydrolysis diffusion method ${ }^{57}$. The nitrate nitrogen (NN), ammonium nitrogen (AN), and available phosphorus (AP) were determined using a continuous flow analyzer (AutoAnalyzer 3 , Bran+Luebbe, Germany). Available potassium (AK) was determined by flame Photometry (FP8800, Topac Inc., MA, USA) and total sulfur (TS) were determined by combustion (CNS-2000; LECO, St. Joseph, MI, USA). Exchangeable calcium (ECa), exchangeable magnesium (EMg) were determined by atomic absorption spectrophotometry (Hitachi Z-5000 Polarized Zeeman, Tokyo, Japan). The microelements, including total ferric iron (TFe), available Ferrous iron (AFe), available molybdenum (AMo), available boron $(A B)$, available zinc (AZn), available copper $(A C u)$, and available manganese (AMn) were determined by Inductively Coupled Plasma Optical Emission Spectrometer (ICP-OES; Agilent, Santa Clara, CA 95051, USA).

\section{DNA extraction and sequencing}

The genomic DNA was extracted from each sample of rhizosphere soil at The Beijing Genomics Institute (BGI) Co., Ltd (BGI-Shenzhen, Guangdong, China). The sequencing library construction method was optimized based on the lllumina operating instructions. The Covaris focused ultrasound system was used to first break the qualified DNA sample. After the end repair was performed by adding End Repair Mix at $20^{\circ} \mathrm{C}$ for 30 minutes, the DNA fragment was purified using a PCR purification kit (QIAGEN, Shanghai, China). A base was added to the $3^{\prime}$-end using A-Tailing Mix at $37^{\circ} \mathrm{C}$ for 30 minutes and then a sequencing adapter was ligated to both ends of the DNA fragment. Fragment selection was performed using 2\% agarose gel and QIA quick Gel Extraction kit (QIAGEN), and several cycles of PCR amplification were performed. The PCR product was again purified with $2 \%$ agarose gel and the target fragment was recovered using an extraction kit (QIAGEN). Finally, the quality of sample library was controlled and quantified using the Agilent 2100 Bioanaylzer and the ABI StepOne Plus Real-Time PCR System. The qualified library was sequenced using an Illumina HiSeq ${ }^{\mathrm{TM}} 2000$ next-generation sequencer ${ }^{58,59}$ at the BGI facility. 
The raw reads produced by the Illumina HiSeq $^{\text {TM }} 2000$ were filtered into clean reads by removing the unqualified reads, which contain more than $10 \%$ unknown bases, 15 or more bases aligned to the adaptor sequence, and more than $20 \%$ of bases in the total reads having a quality score lower than 15 after sequencing.

\section{Bioinformatics analysis}

The qualified reads were first assembled by using SOAP denovo $2^{60}$ and Rabbit ${ }^{61}$. Gene predictions were then performed using the MetaGeneMark ${ }^{62}$. After the predicted genes were clustered with Cd-hit, a nonredundant gene category was obtained ${ }^{63}$. The information of gene function and species were gained by the gene category mapping to the public databases, including NR, Swiss-Prot, KEGG ${ }^{64}$, and gene ontology $(\mathrm{GO})^{65}$. The reads were then aligned against the gene category, and the gene and species abundance of each sample was calculated. Finally, species diversity analysis (Shannon index and comparative analysis), principle component analysis (PCA), GO functional classification analysis, and pathway enrichment analysis performed based on the gene and species abundance data.

For metagenomics de novo assembly of each sample, the different k-mer sizes were used to perform multiple de novo assembly at the same time and reads then compared with the assembly results to evaluate the assembled effect. Finally, comprehensively to consider the median length of fragments assembled (N50) and mapping rate, the best assembly result of each sample was selected for subsequent analysis.

The analysis of gene expression was performed by using the Reads per Kilobase per Million reads method (RPKM) ${ }^{66}$. A false discovery rate (FDR) $\leq 0.001^{67}$ and an absolute value of the log (fold-change ratio) $\geq 1$ were set as the threshold to identify the significantly differentially expressed genes (DEGs) between the rhizosphere soil of chlorotic and normal plants.

For annotation, all the DEGs were searched against the National Centre for Biotechnology Information (NCBI) for non-redundant protein (NR), Swiss-Prot, EuKaryotic Orthologous Groups of proteins (KOG) (Altschul et al. 1990), and GO databases with a BLAST alignment. The pathway enrichment analysis of DEGs was completed by using Kyoto Encyclopedia of Genes and Genomes ${ }^{68}$ All the standard bioinformatics analyses were carried out at the BGI facility.

\section{Field investigation and pathogen isolation}

The absorption roots in the upper, middle, and lower layer of soil were sampled for the pathogen isolations of fungal and bacterial diseases according to reported protocols 37,69 .

\section{Statistical analysis}


Primary data from the soil analyses and relative abundance of bacteria species were subjected to oneway analysis of variance (ANOVA). When differences were found at $P \leq 0.05$ or $P \leq 0.01$, least significant difference (LSD) values were calculated to compare the means using Duncan's multiple range test in SPSS Version 19.0 software for Windows (SPSS Inc., Chicago, IL, USA).

\section{Declarations}

\section{Acknowledgments}

This project was financially supported by the earmarked fund for the China Agriculture Research System (CARS-28-14), the China National Key Research \& Development Program (2018YFD02014), and Excellent Young Talents Fund Program of Higher Education Institutions of Anhui Province (gxfxZD201602).

\section{Author information}

\section{Affiliations}

School of Horticulture, Anhui Agricultural University, Hefei 230036, Anhui, P.R. China

Bing Jia, Xiao Chang, Yuanyuan Fu, Wei Heng, Zhenfeng Ye, Pu Liu, Li Liu, \& Liwu Zhu

College of Agriculture and Life Sciences, Cornell University, Ithaca, NY 14853, USA

Yosef Al Shoffe \& Christopher Brian Watkins

\section{Contributions}

BJ performed the data analysis and drafted the manuscript. XC and YYF sampled the soil material and submitted soil for minerals measurement. ZFY, WH and YAS assisted in bioinformatic and statistical analysis of the data. LWZ was supervisors for experiment design and coordination, written and revised the manuscript. PL participated in part of the experiment design. CBW provided suggestions and language revisions of the manuscript. All authors commented on previous versions of the manuscript and have read and approved the final manuscript.

\section{Corresponding authors}

Correspondence to Liwu Zhu.

\section{Ethics declarations}

\section{Competing interests}

The authors declare that they have no competing interests of this paper.

\section{References}


1. FAOSTAT http://www.fao.org/faostat/en/\#data/QC (2018).

2. Briat, J.F., Dubos, C. \& Gaymard, F. Iron nutrition, biomass production, and plant product quality. Trends Plant Sci.20, 33-40 (2015).

3. Abadía, J. et al. Technologies for the diagnosis and remediation of Fe deficiency. Soil Sci. Plant Nutr. 50, 965-971 (2004).

4. Obreza, T.A., Zekric, M. \& Calvert, D.V. Citrus fertilizer management on calcareous soils. UF/IFAS, CIR. 1127 (1993).

5. Kannan, S. Occurrence of iron deficiency in important crops in maharashtra and tamil nadu states in India: a report. J Plant Nutr.11, 1285-1293 (1988).

6. Korcak, R.F. Iron deficiency chlorosis. Hort Rev. 9, 133-186 (1987).

7. Amoros. J.A. et al. Iron uptake in vineyard soils and relationships with other elements ( $\mathrm{Zn}, \mathrm{Mn}$ and Ca). The case of Castilla-La Mancha, Central Spain. App/ Geochem.88, 17-22 (2018).

8. Bravo, S. et al. Influence of the soil pH in the uptake and bioaccumulation of heavy metals (Fe, Zn, $\mathrm{Cu}, \mathrm{Pb}$ and $\mathrm{Mn}$ ) and other elements ( $\mathrm{Ca}, \mathrm{K}, \mathrm{Al}, \mathrm{Sr}$ and $\mathrm{Ba}$ ) in vine leaves, Castilla-La Mancha (Spain). J Geochem Explor. 174, 79-83 (2017).

9. Briat, J.F. \& Gaymard, F. Iron utilization and metabolism in plants. Curr opin plant boil.10, 276-282 (2007).

10. Jeong. J. \& Guerinot, M.L. Homing in on iron homeostasis in plants. Trends Plant Sci. 14, 280-285 (2009).

11. McNear, D.H.; Jr. The rhizosphere-roots, soil and everything in between. Nature Education Knowledge 4, 1 (2013).

12. Juan, L.J.; Hernandez-Apaolaza, L. Iron nutrition in plants: an overview. Plant Soil. 418, 1-4 (2017).

13. Grotz, N, \& Guerinot, M.L. Molecular aspects of $\mathrm{Cu}$, Fe and $\mathrm{Zn}$ homeostasis in plants. Biochimi Biophys Acta. 1763, 595-608 (2006).

14. Chaparro, J.M.; Sheflin, A.M.; Manter, D.K.; Vivanco, J.M. Manipulating the soil microbiome to increase soil health and plant fertility. Bio Fert Soils.48, 489-499 (2012).

15. Turner, T.R. et al. Comparative metatranscriptomics reveals kingdom level changes in the rhizosphere microbiome of plants. ISME J.7, 2248-2258 (2013).

16. Philippot, L., Raaijmakers, J.M., Lemanceau, P., Van, D.P. \& Wim, H. Going back to the roots: the microbial ecology of the rhizosphere. Nat Rev Microbiol.11, 789-799 (2013).

17. Bulgarelli, D., Schlaeppi, K., Spaepen, S., Van-Themaat, EV.L. \& Schulze-Lefert, P. Structure and functions of the bacterial microbiota of plants. Annu Rev of Plant Biol. 64, 807-838 (2013).

18. Bulgarelli, D. et al. Structure and function of the bacterial root microbiota in wild and domesticated barley. Cell Host Microbe. 17, 392-403 (2015).

19. Jacoby, R., Peukert, M., Succurro, A., Koprivova, A. \& Kopriva, S. The role of soil microorganisms in plant mineral nutrition-current knowledge and future directions. Front Plant Sci.8, 1617 (2017). 
20. Fitzpatricka, C.R. et al. Assembly and ecological function of the root microbiome across angiosperm plant species. Proc Natl Acad Sci U S A. 115, e1157-e1165 (2018).

21. Spence, C. \& Bais, H. Probiotics for plants: Rhizospheric microbiome and plant fitness. Mol Microb Ecol Rhizosphere.12, 713-721 (2013).

22. Hou, M.P. \& Babalola, O.O. Evaluation of plant growth promoting potential of four rhizobacterial species for indigenous system. J Cent South Univ.20, 164-171 (2013).

23. Chaparro, J.M.; Badri, D.V.; Vivanco, J.M. Rhizosphere microbiome assemblage is affected by plant development. ISME J. 8, 790-803 (2014).

24. Massalha, H., Korenblum, E., Tholl, D. \& Aharoni, A. Small molecules below-ground: the role of specialized metabolites in the rhizosphere. Plant J. 90, 788-807 (2017).

25. Naylor, D., DeGraaf, S., Purdom, E. \& Coleman-Derr, D. Drought and host selection influence bacterial community dynamics in the grass root microbiome. ISME J.11, 2691-2704 (2017).

26. Santos-Medellín, C., Edwards, J., Liechty, Z., Nguyen, B. \& Sundaresan, V. Drought stress results in a compartment-specific restructuring of the rice root-associated microbiomes. Mbio.8, e00764-17 (2017).

27. Bouffaud, M.L. et al. Is diversification history of maize influencing selection of soil bacteria by roots?. Mol Ecol.21, 195-206 (2012).

28. Peiffer, J.A. et al. Diversity and heritability of the maize rhizosphere microbiome under field conditions. Proc Natl Acad Sci U S A. 110, 6548-6553 (2013).

29. Pérez-Jaramillo, J.E., Mendes, R. \& Raaijmakers, J.M. Impact of plant domestication on rhizosphere microbiome assembly and functions. Plant Mol Biol.90, 635-644 (2016).

30. Qiao, Q.H. et al. The variation in the rhizosphere microbiome of cotton with soil type, genotype and developmental stage. Sci Rep-UK. 7, 3940 (2017).

31. Fernández, V. et al. Leaf structural changes associated with iron deficiency chlorosis in field-grown pear and peach: physiological implications. Plant Soil.311, 161-172 (2008).

32. Lopez-Millan, A.F., Millan, F., Abadia, A. \& Abadia, J. Effects of iron deficiency on the composition of the leaf apoplastic fluid and xylem sap in sugar beet. Implications for iron and carbon transport. Plant Physiol. 124, 873-884 (2000).

33. Nouet, C., Motte, P. \& Hanikenne, M. Chloroplastic and mitochondrial metal homeostasis. Trends Plant Sci. 16, 395-404 (2011).

34. Hansch, R. \& Mendel, R.R. Physiological functions of mineral micronutrients ( $\mathrm{Cu}, \mathrm{Zn}, \mathrm{Mn}, \mathrm{Fe}, \mathrm{Ni}, \mathrm{Mo}, \mathrm{B}$ Cl). Curr Opin Plant Biol. 12, 259-266 (2009).

35. Barton, L.L. \& Abadía, J. Iron nutrition in plants and rhizospheric microorganisms. Springer Verlag. 6, $1-22(2006)$.

36. Alvarez-Fernandez, A., Melgar, J.C., Javier, A. \& Anunciacion, A. Effects of moderate and severe iron deficiency chlorosis on fruit yield, appearance and composition in pear (Pyrus communis L.) and peach (Prunus persica (L.) Batsch). Environ Exp Bot.71, 280-286 (2011). 
37. Zhu, L.W. et al. First Report of Fusarium root rot in Asian Pear caused by Fusarium solani in China. Plant Dis.101, 252 (2016).

38. Van, K. et al. Complete nitrification by a single microorganism. Nature.528, 555-559 (2015).

39. Daims, H. et al. Complete nitrification by nitrospira bacteria. Nature. 528, 504-509 (2015).

40. Spieck. E., Hartwig, C., McCormack, I., Maixner, F. \& Daims, H. Selective enrichment and molecular characterization of a previously uncultured Nitrospira -like bacterium from activated sludge. Environ Microbio.8, 405-415 (2006).

41. Nowka, Boris., Holger, D. \& Eva, S. Comparison of oxidation kinetics of nitrite-oxidizing bacteria: nitrite availability as a key factor in niche differentiation. Appl Environ Microbiol.81, 745-753 (2015).

42. DeBruyn, J.M., Nixon, L.T., Fawaz, M.N., Johnson, A.M. \& Radosevich, M. Global biogeography and quantitative seasonal dynamics of gemmatimonadetes in soil. Appl Environ Microb. 77, 6295-6300 (2011).

43. DeBruyn, J.M. et al. Genome sequence and methylome of soil bacterium Gemmatirosa kalamazoonensis KBS708T, a member of the rarely cultivated Gemmatimonadetes phylum. Genome A.2, e00226-14 (2014).

44. DeBruyn, J.M. et al. Gemmatirosa kalamazoonensis gen. nov., sp. nov., a member of the rarelycultivated bacterial phylum Gemmatimonadetes. J Gen Appl Microb. 59, 305-312 (2013).

45. Kojima, H. \& Fukui, M. Sulfuricella denitrificans gen. nov., sp. nov., a sulfur-oxidizing autotroph isolated from a freshwater lake. Int J Syst Evol Micr. 60, 2862-2866 (2010).

46. Watanabe, T., Kojima, H. \& Fukui, M. Complete genomes of freshwater sulfur oxidizers Sulfuricella denitrificans skB26 and Sulfuritalea hydrogenivorans sk43H: Genetic insights into the sulfur oxidation pathway of betaproteobacteria. Syst App/ Microbiol. 37, 387-395 (2014).

47. Zhang, H. et al. Gemmatimonas aurantiaca gen. nov., sp. nov., a gram-negative, aerobic, polyphosphate accumulating micro-organism, the first cultured representative of the new bacterial phylum Gemmatimonadetes phyl. nov. Int J Syst Evol Microbiol. 53, 1155-1163 (2003).

48. Joseph, S.J., Hugenholtz, P., Sangwan, P., Osborne, C.A. \& Janssen, P.H. Laboratory cultivation of widespread and previously uncultured soil bacteria. Appl Environ Microb. 69, 7210-7215 (2003).

49. Ward, N.L. et al. Three genomes from the phylum Acidobacteria provide insight into the lifestyles of these microorganisms in soils. Appl Environ Microbiol.75, 2046-2056 (2009).

50. Davis, K.E., Joseph, S.J. \& Janssen, P.H. Effects of growth medium, inoculum size, and incubation time on culturability and isolation of soil bacteria. Appl Environ Micro. 71, 826-834 (2005).

51. Stearman, R., Yuan, D.S., Yamaguchi-Iwai, Y., Klausner, R.D. \& Dancis, A. A permease-oxidase complex involved in high-affinity iron uptake in yeast. Science.271, 1552-1557 (1996).

52. Kim, B.K. et al. Genome sequence of an ammonia-oxidizing soil archaeon, "candidatus nitrosoarchaeum koreensis" MY1. J Bacteriol. 193, 5539-5540 (2011).

53. Clark, P.D., Pazdernik, J.N. \& McGehee, R.M. Chapter 29 Molecular Evolution, In: Molecular Biology (Third Edition) , Elsevier Inc., Amsterdam. 925-969 (2019). 
54. Woodson, J.D. et al. Ubiquitin facilitates a quality-control pathway that removes damaged chloroplasts. Science. 350, 450-454 (2015).

55. Jackson, M.L. Soil chemical analysis. Prentice Hall Inc., Englewood Chiffs. 213-214 (1958).

56. Chapman, H.D. Cation exchange capacity. In: Page AL (ed) Methods of soil analysis. American Society of Agronomy. Soil Sci Soc Am J.2, 149-157 (1965).

57. Parkinson, J.A. \& Allen, S.E. A wet oxidation procedure suitable for the determination of nitrogen and mineral nutrients in biological material. Commun Soil Sci Plant An. 6, 1-11 (1975).

58. Caporaso, J.G. et al. Global patterns of $16 \mathrm{~S}$ rRNA diversity at a depth of millions of sequences per sample. Proc Natl Acad Sci U S A. 108, 4516-4522 (2011).

59. Caporaso, J.G. et al. Ultra-high-throughput microbial community analysis on the Illumina HiSeq and MiSeq platforms. The ISME J.6, 1621-1624 (2012).

60. Luo, R.R. et al. SOAPdenovo2: an empirically improved memory-efficient short-read de novo assembler. Gigascience. 27, 18 (2012).

61. You, M.S. et al.. A heterozygous moth genome provides insights into herbivory and detoxification. Nat Genet.45, 220-225 (2013).

62. Zhu, W., Lomsadze, A. \& Borodovsky, M. Ab initio gene identification in metagenomic sequences. Nucleic Acids Res. 38, e132 (2010).

63. Li, W.Z. \& Godzik, A. Cd-hit: a fast program for clustering and comparing large sets of protein or nucleotide sequences. Bioinformatics.22, 1658-1659 (2006).

64. Altschul, S.F.; Gis, W.; Miller, W.; Myers, E.W.; Lipman, D.J. Basic local alignment search tool. J. Mol. Biol. 215, 403-410 (1990).

65. Conesa, A. et al. Blast2GO: a universal tool for annotation, visualization and analysis in functional genomics research. Bioinformatics.21, 3674-3676 (2005).

66. Mortazavi, A. et al. Mapping and quantifying mammalian transcriptomes by RNASeq. Nat Methods. 5, 621-628 (2008).

67. Audic, S. \& Claverie, J.M. The significance of digital gene expression profiles. Genome Res.7, 986995 (1997)..

68. Kanehisa, M . et al. KEGG for linking genomes to life and the environment. Nucleic Acids Res.36, D480-D484 (2008).

69. He, R. et al. Genetic diversity of pseudomonas syringae pv. actinidiae strains from different geographic regions in china. Phytopathology. 109, 347-357 (2019).

\section{Figures}



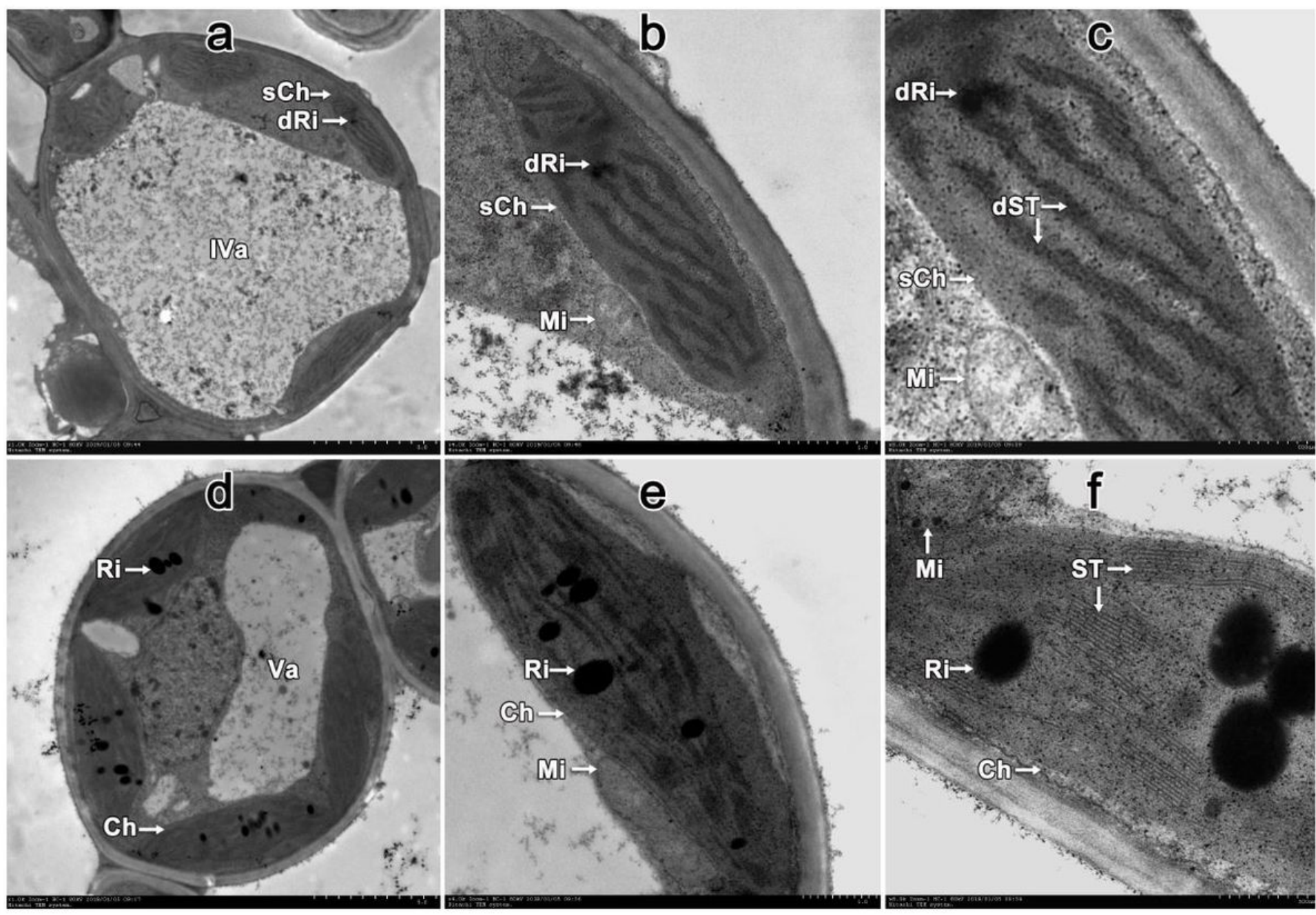

\section{Figure 1}

Subcellular structure of leaf from chlorotic and normal plants observed by transmission electron microscope Degraded ribosome (dRi), destroyed stack of thylakloid (dST) in smaller chloroplast (sCh), larger vacuole (IVa), and normal mitochondria (Mi) found in the cell of leaf with severe Fe-deficiency from chlorotic plants (a, b, and c). Normal ribosome (Ri), stack of thylakloid (ST), chloroplast (Ch), vacuole (Va) and Mi observed in the cell of leaf from normal plants ( $d$, e, and $f)$. The magnifications $(\times 1.0 \mathrm{k}, \times 4.0 \mathrm{k}$, and $8.0 \mathrm{k})$ and scale bars $(5 \mu \mathrm{m}, 1 \mu \mathrm{m}$ and $500 \mathrm{~nm})$ were at the bottom of each panel, respectively. 


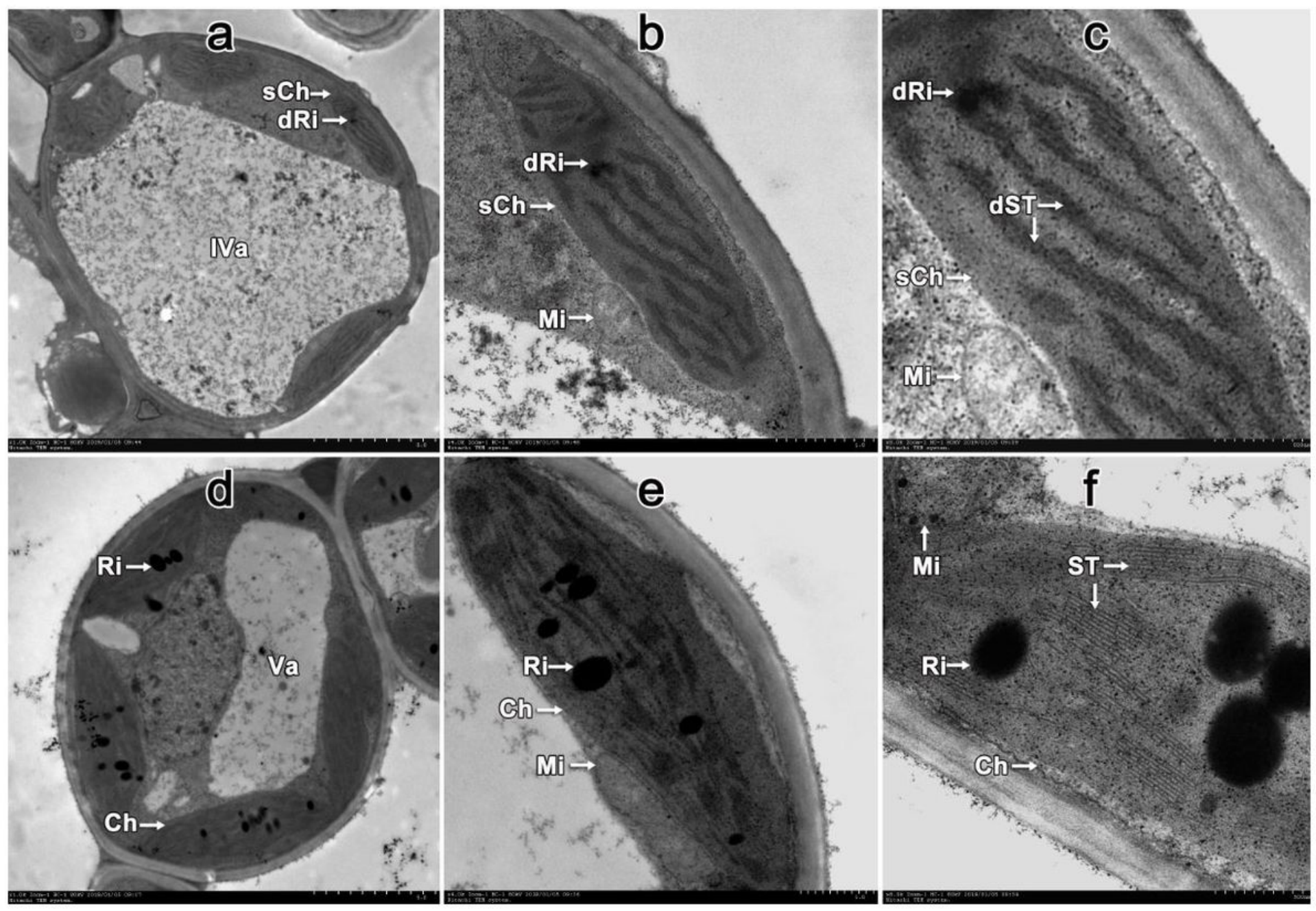

\section{Figure 1}

Subcellular structure of leaf from chlorotic and normal plants observed by transmission electron microscope Degraded ribosome (dRi), destroyed stack of thylakloid (dST) in smaller chloroplast (sCh), larger vacuole (IVa), and normal mitochondria (Mi) found in the cell of leaf with severe Fe-deficiency from chlorotic plants (a, b, and c). Normal ribosome (Ri), stack of thylakloid (ST), chloroplast (Ch), vacuole (Va) and Mi observed in the cell of leaf from normal plants ( $d$, e, and $f)$. The magnifications $(\times 1.0 \mathrm{k}, \times 4.0 \mathrm{k}$, and $8.0 \mathrm{k})$ and scale bars $(5 \mu \mathrm{m}, 1 \mu \mathrm{m}$ and $500 \mathrm{~nm})$ were at the bottom of each panel, respectively. 

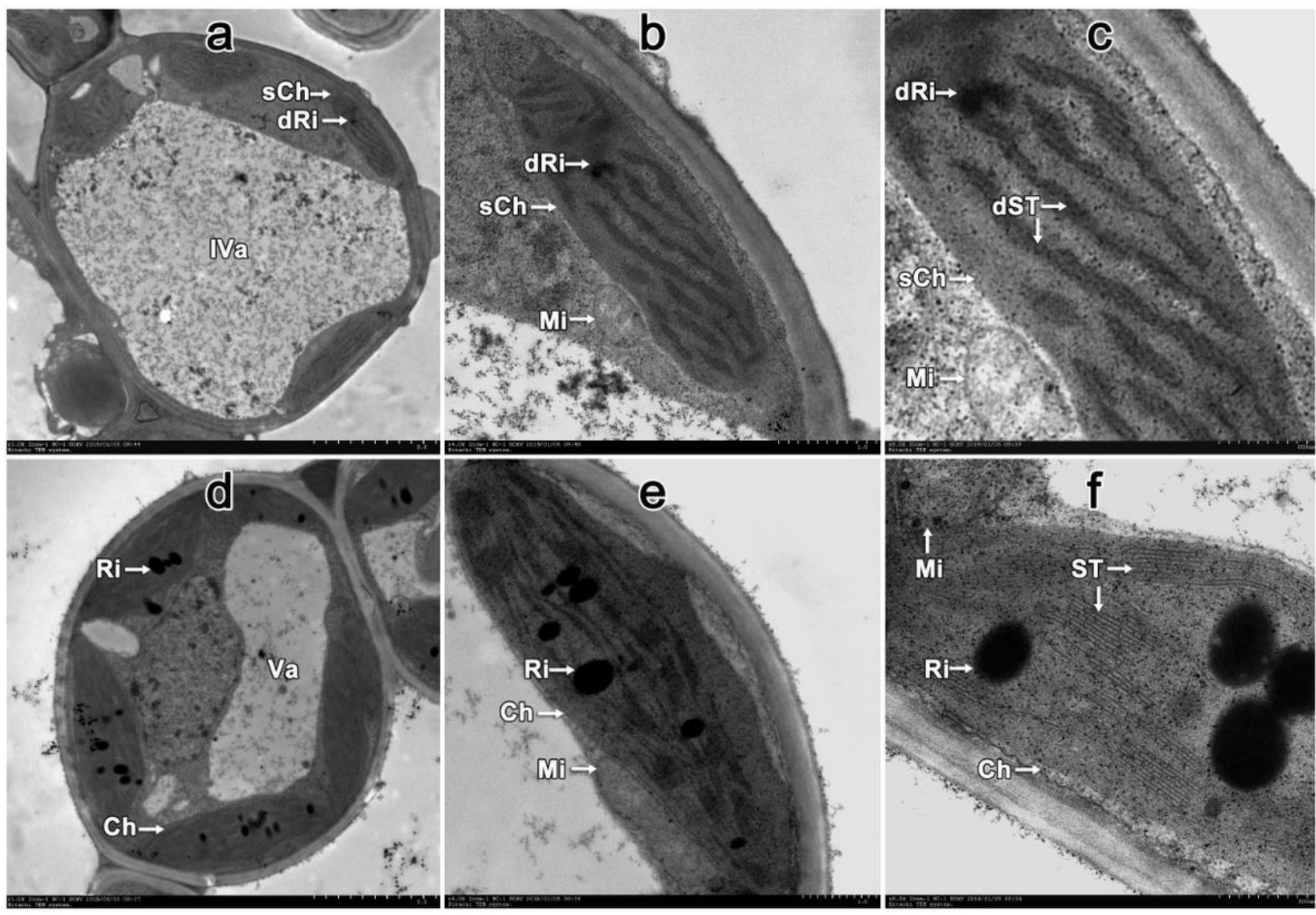

\section{Figure 1}

Subcellular structure of leaf from chlorotic and normal plants observed by transmission electron microscope Degraded ribosome (dRi), destroyed stack of thylakloid (dST) in smaller chloroplast (sCh), larger vacuole (IVa), and normal mitochondria (Mi) found in the cell of leaf with severe Fe-deficiency from chlorotic plants ( $\mathrm{a}, \mathrm{b}$, and $\mathrm{c})$. Normal ribosome (Ri), stack of thylakloid (ST), chloroplast (Ch), vacuole (Va) and Mi observed in the cell of leaf from normal plants ( $d$, e, and $f)$. The magnifications $(\times 1.0 \mathrm{k}, \times 4.0 \mathrm{k}$, and $8.0 \mathrm{k})$ and scale bars $(5 \mu \mathrm{m}, 1 \mu \mathrm{m}$ and $500 \mathrm{~nm})$ were at the bottom of each panel, respectively. 

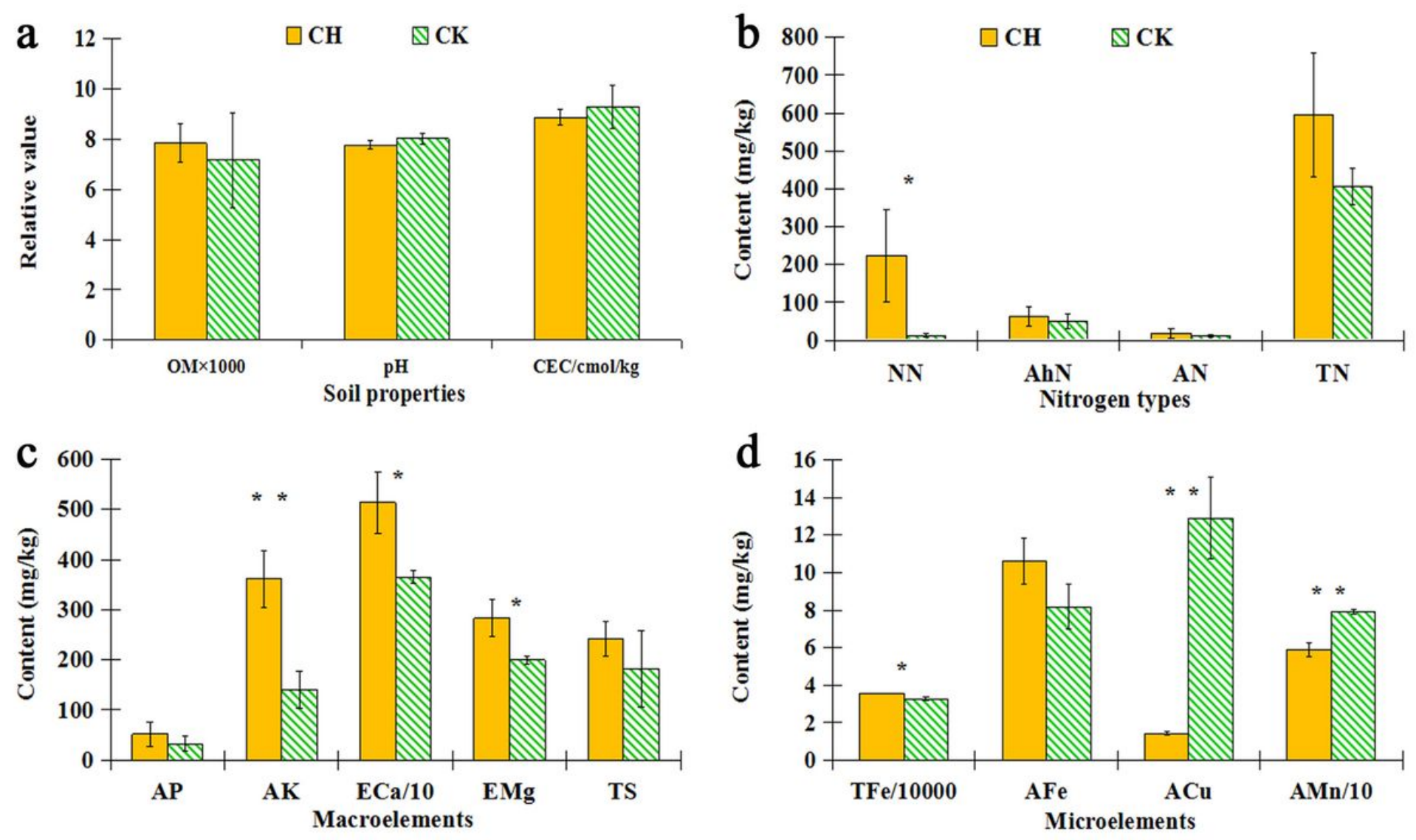

Figure 2

Physiochemical properties in the rhizosphere soil of Fe-deficiency chlorosis $(\mathrm{CH})$ and normal plants (CK) a. Organic matter (OM), pH value, cation exchange capacity (CEC), total ferrum (TFe), and available ferrous (Fe2+) contents. b. Nitrate nitrogen (NN), alkali hydrolysable nitrogen ( $\mathrm{AhN}$ ), amonium nitrogen (AN), and total nitrogen (TN) contents. c. Available phosphorus (AP), available potassium (AK), exchangeable calcium (ECa), exchangeable magnesium (EMg), and total sulfur (TS) content. d. Available microelements (Mo, $B, \mathrm{Zn}, \mathrm{Cu}$, and $\mathrm{Mn}$ ). Error bars indicate \pm standard error ( $\mathrm{SE}, \mathrm{n}=3)$, * $P$-value $\leq 0.05$, and $* *$ P-value $\leq 0.01$. 

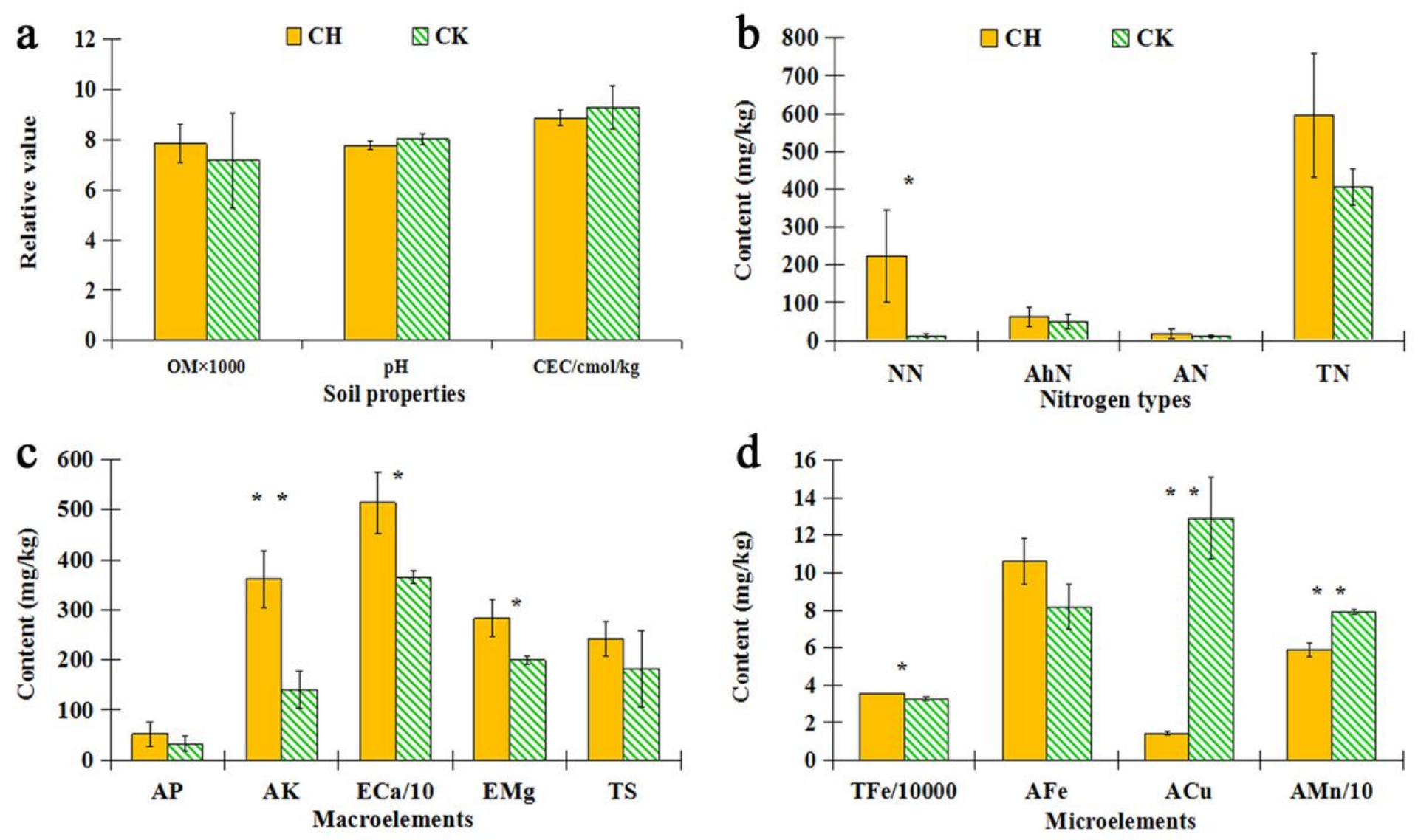

Figure 2

Physiochemical properties in the rhizosphere soil of Fe-deficiency chlorosis $(\mathrm{CH})$ and normal plants (CK) a. Organic matter (OM), pH value, cation exchange capacity (CEC), total ferrum (TFe), and available ferrous (Fe2+) contents. b. Nitrate nitrogen (NN), alkali hydrolysable nitrogen ( $\mathrm{AhN}$ ), amonium nitrogen (AN), and total nitrogen (TN) contents. c. Available phosphorus (AP), available potassium (AK), exchangeable calcium (ECa), exchangeable magnesium (EMg), and total sulfur (TS) content. d. Available microelements (Mo, $B, \mathrm{Zn}, \mathrm{Cu}$, and $\mathrm{Mn}$ ). Error bars indicate \pm standard error ( $\mathrm{SE}, \mathrm{n}=3)$, * $P$-value $\leq 0.05$, and $* *$ P-value $\leq 0.01$. 

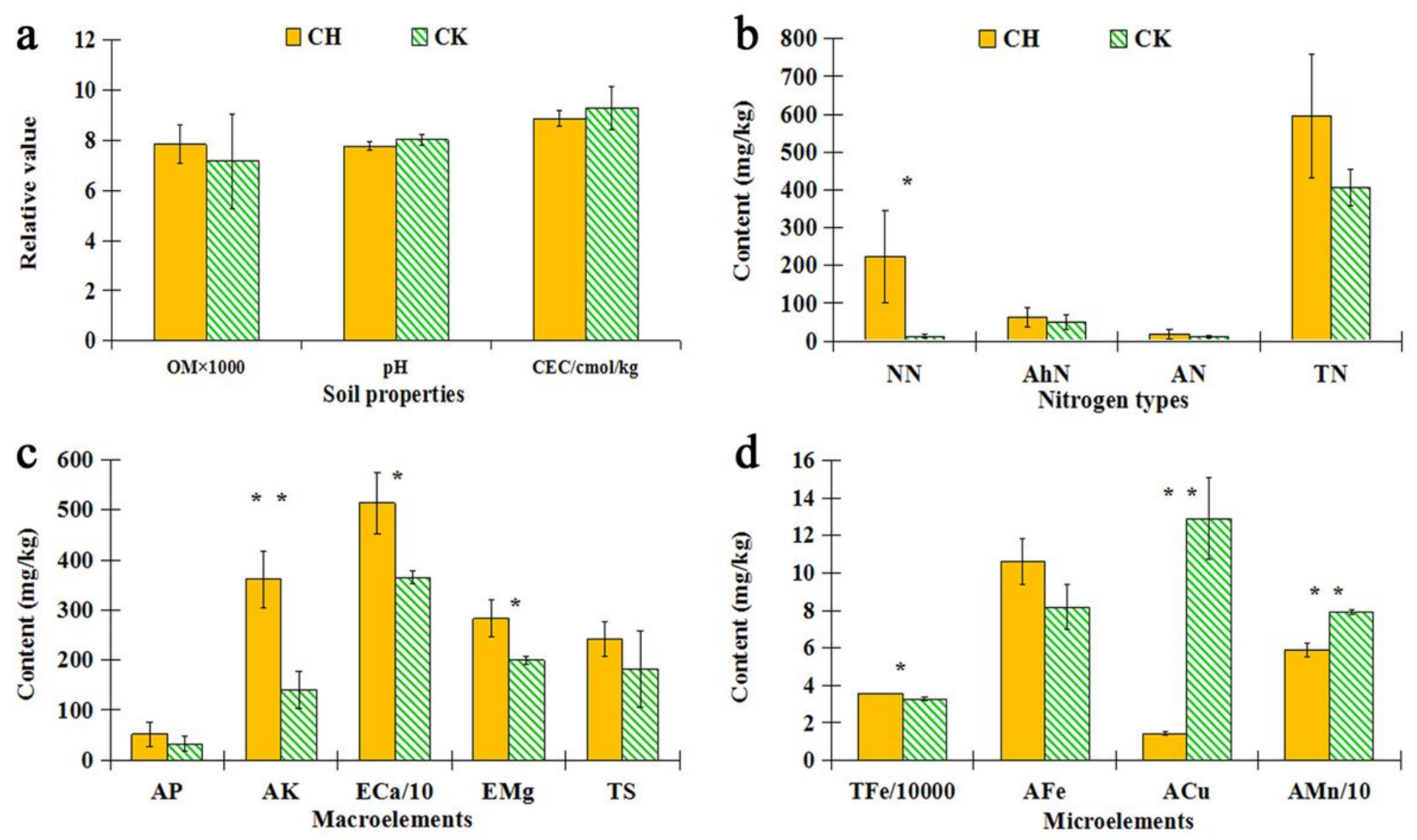

Figure 2

Physiochemical properties in the rhizosphere soil of Fe-deficiency chlorosis $(\mathrm{CH})$ and normal plants (CK) a. Organic matter (OM), pH value, cation exchange capacity (CEC), total ferrum (TFe), and available ferrous (Fe2+) contents. b. Nitrate nitrogen (NN), alkali hydrolysable nitrogen ( $\mathrm{AhN}$ ), amonium nitrogen (AN), and total nitrogen (TN) contents. c. Available phosphorus (AP), available potassium (AK), exchangeable calcium (ECa), exchangeable magnesium (EMg), and total sulfur (TS) content. d. Available microelements (Mo, $B, \mathrm{Zn}, \mathrm{Cu}$, and $\mathrm{Mn}$ ). Error bars indicate \pm standard error ( $\mathrm{SE}, \mathrm{n}=3)$, * $P$-value $\leq 0.05$, and $* *$ P-value $\leq 0.01$. 


\section{GO Functional Classification \\ (CK-VS-CH.GeneDiffAbunFilter.xls)}

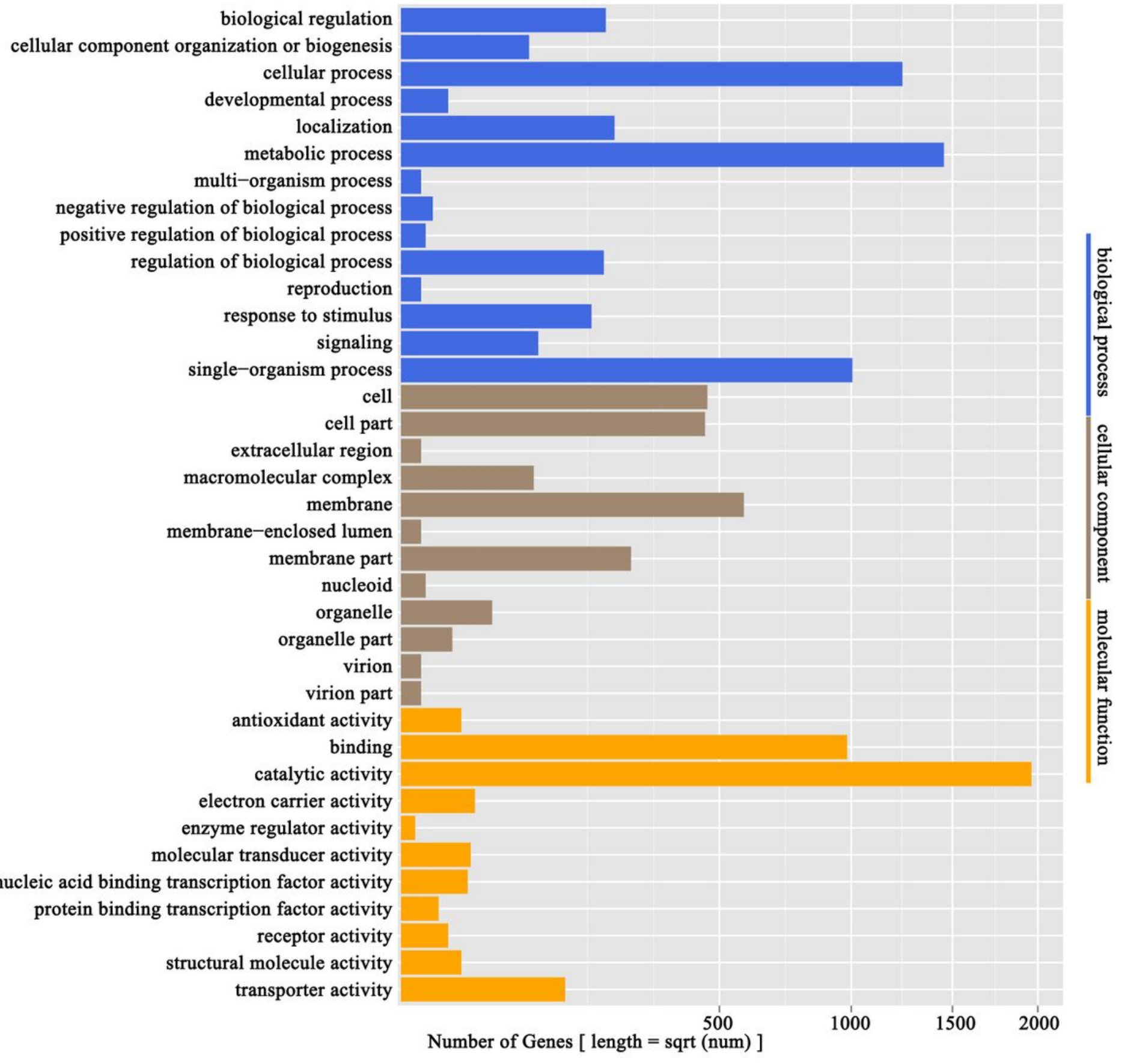

\section{Figure 3}

GO functional classification of differently expressed genes between the rhizosphere soil of chlorosis and normal plants. $\mathrm{CH}$. rhizosphere soil of chlorotic plants; $\mathrm{CK}$. rhizosphere soil of normal plant. 


\section{GO Functional Classification \\ (CK-VS-CH.GeneDiffAbunFilter.xls)}

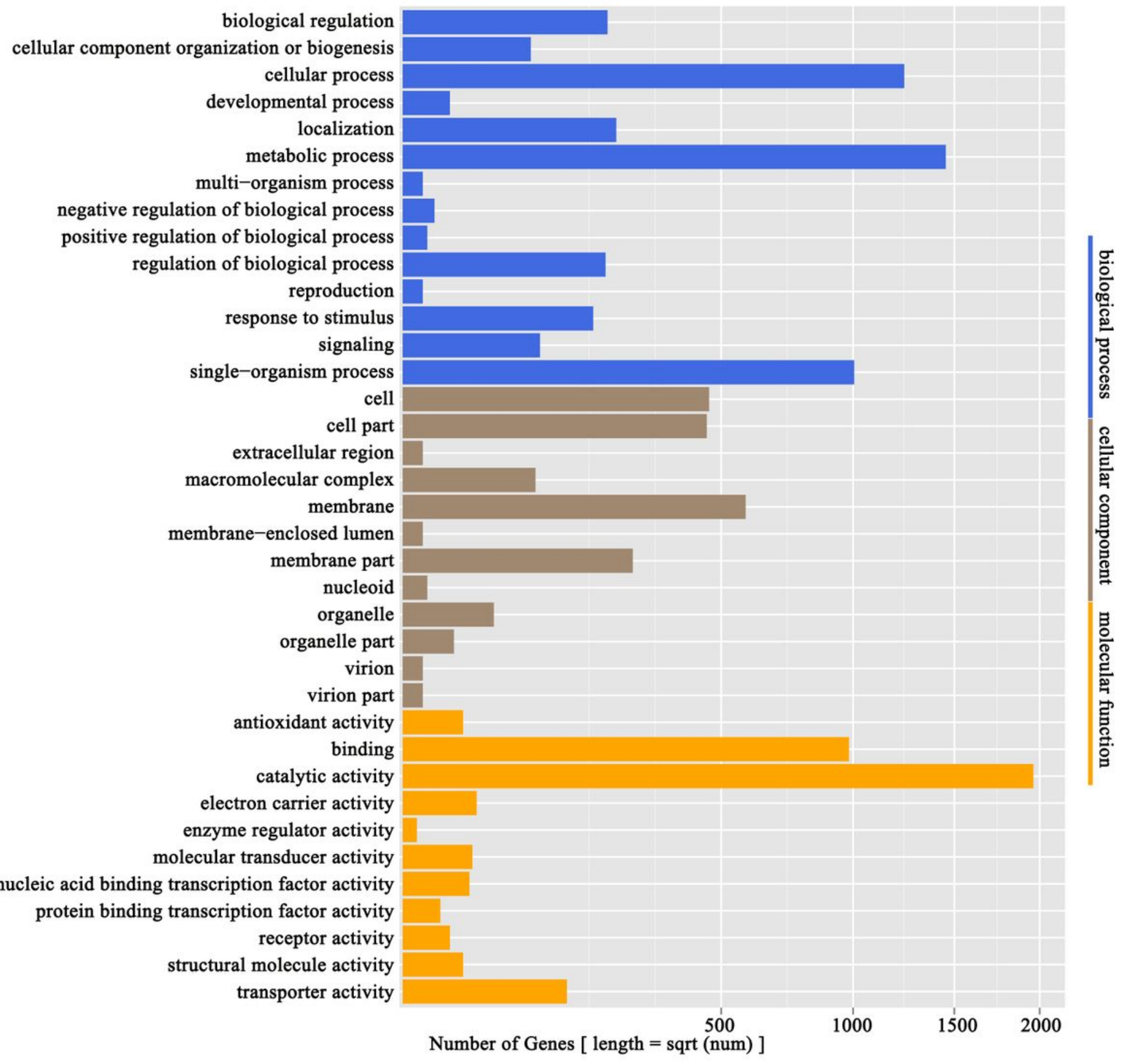

\section{Figure 3}

GO functional classification of differently expressed genes between the rhizosphere soil of chlorosis and normal plants. $\mathrm{CH}$. rhizosphere soil of chlorotic plants; $\mathrm{CK}$. rhizosphere soil of normal plant. 


\section{GO Functional Classification \\ (CK-VS-CH.GeneDiffAbunFilter.xls)}

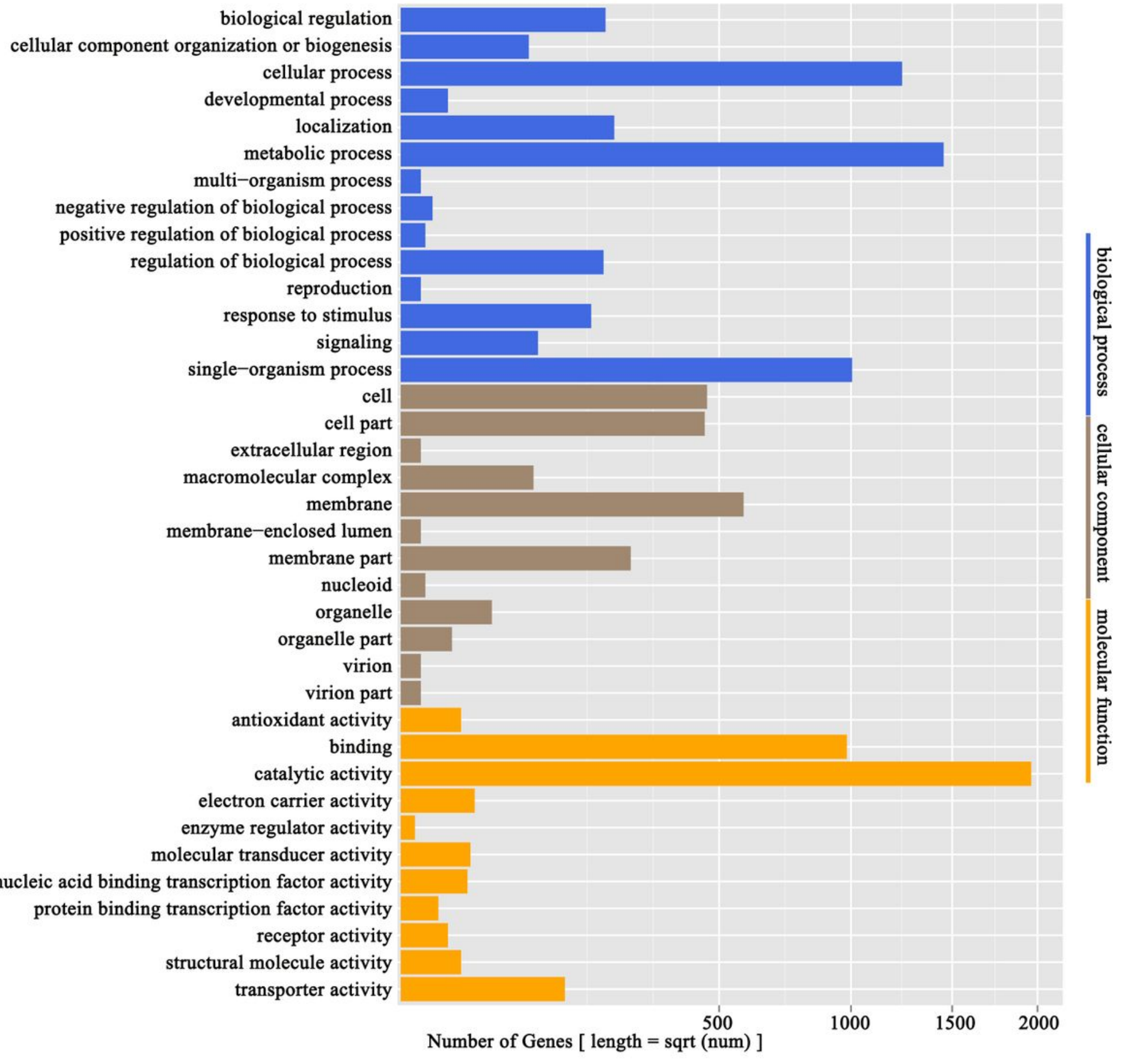

\section{Figure 3}

GO functional classification of differently expressed genes between the rhizosphere soil of chlorosis and normal plants. $\mathrm{CH}$. rhizosphere soil of chlorotic plants; CK. rhizosphere soil of normal plant. 


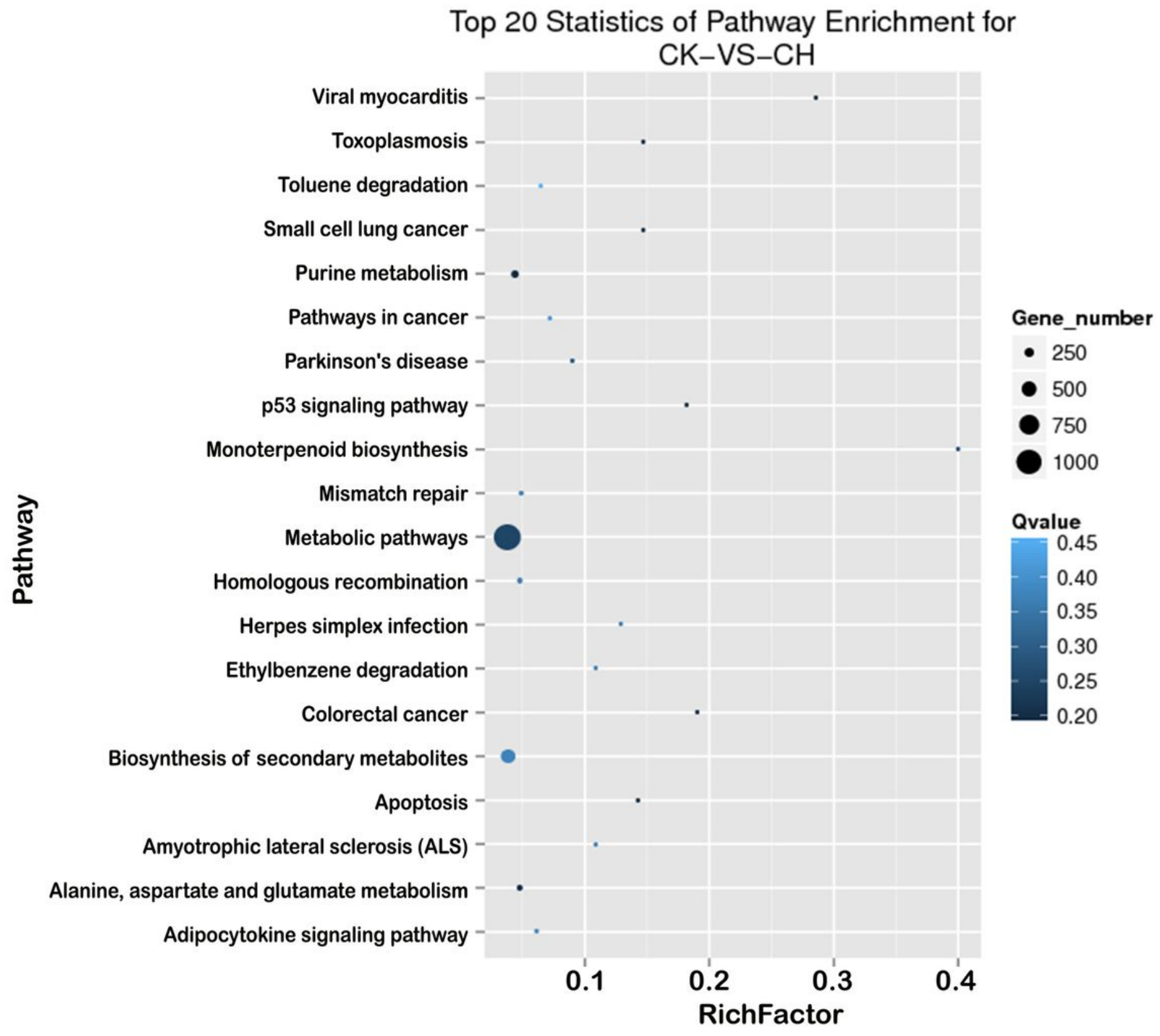

\section{Figure 4}

Top 20 pathways enriched for the differently expressed genes between rhizosphere soil of chlorosis and normal plants. CK1-3. rhizosphere soil of chlorotic plants; $\mathrm{CH1}$-3. rhizosphere soil of normal plants. 


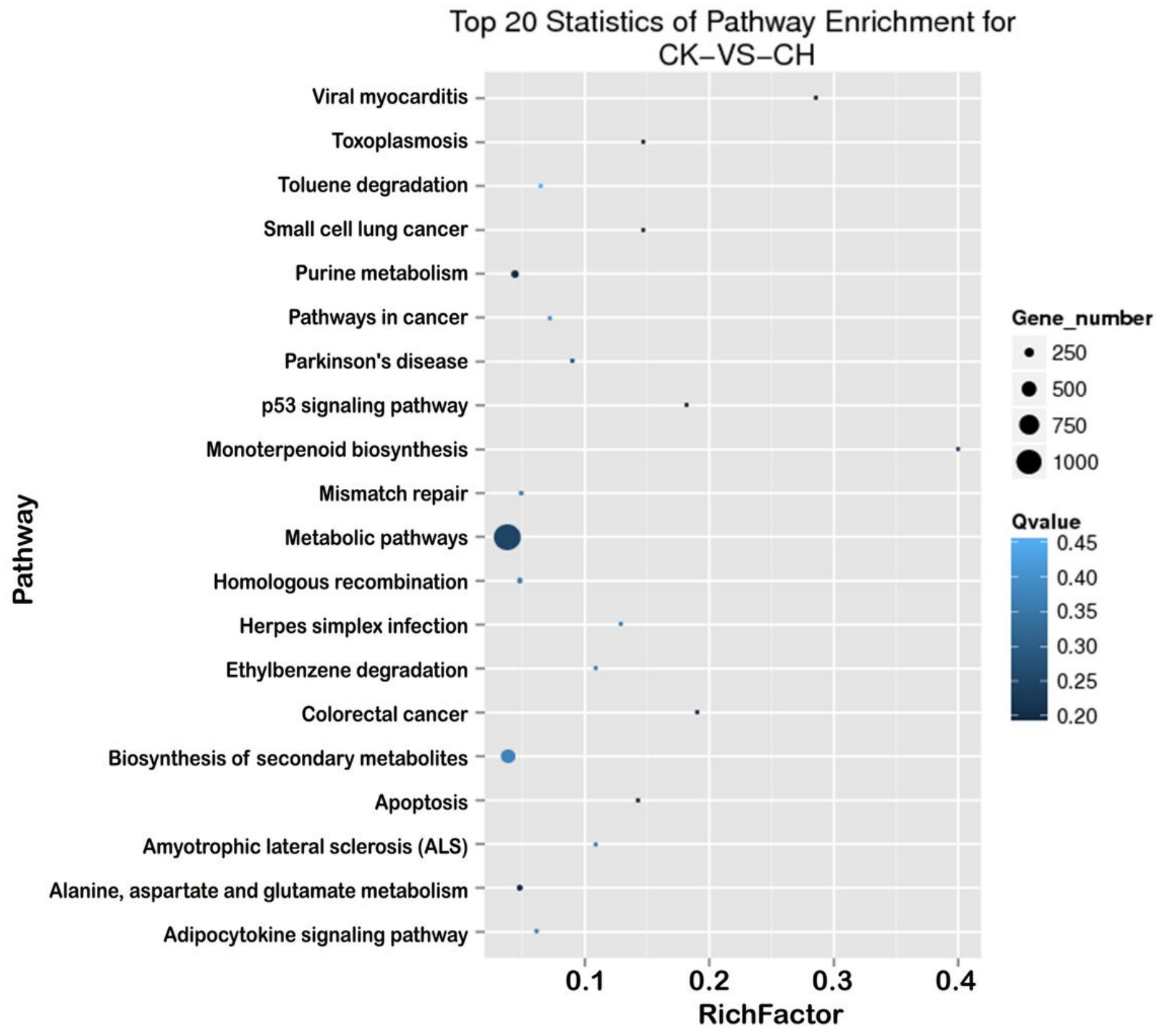

\section{Figure 4}

Top 20 pathways enriched for the differently expressed genes between rhizosphere soil of chlorosis and normal plants. CK1-3. rhizosphere soil of chlorotic plants; $\mathrm{CH1}$-3. rhizosphere soil of normal plants. 


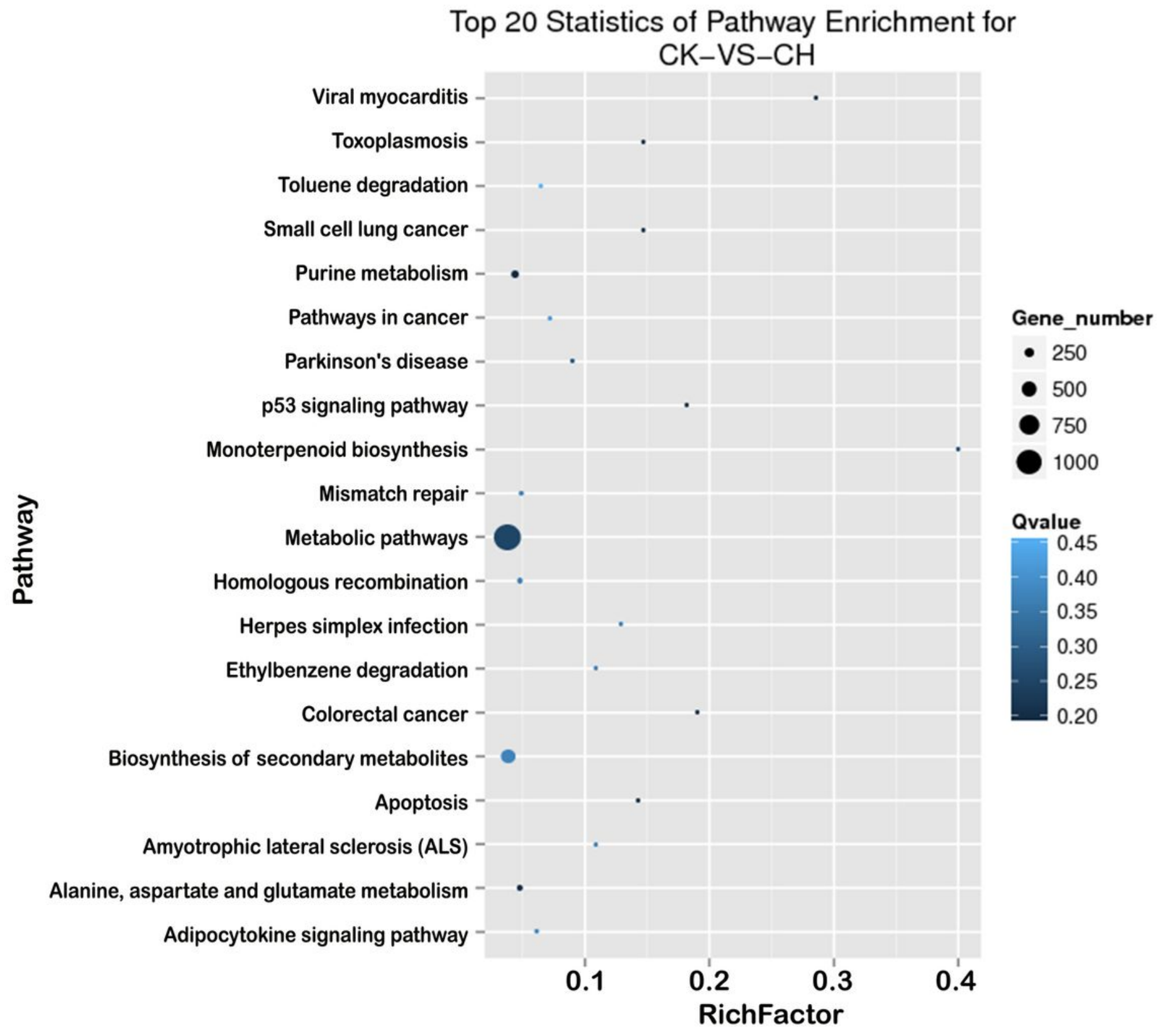

\section{Figure 4}

Top 20 pathways enriched for the differently expressed genes between rhizosphere soil of chlorosis and normal plants. CK1-3. rhizosphere soil of chlorotic plants; $\mathrm{CH1}$-3. rhizosphere soil of normal plants. 


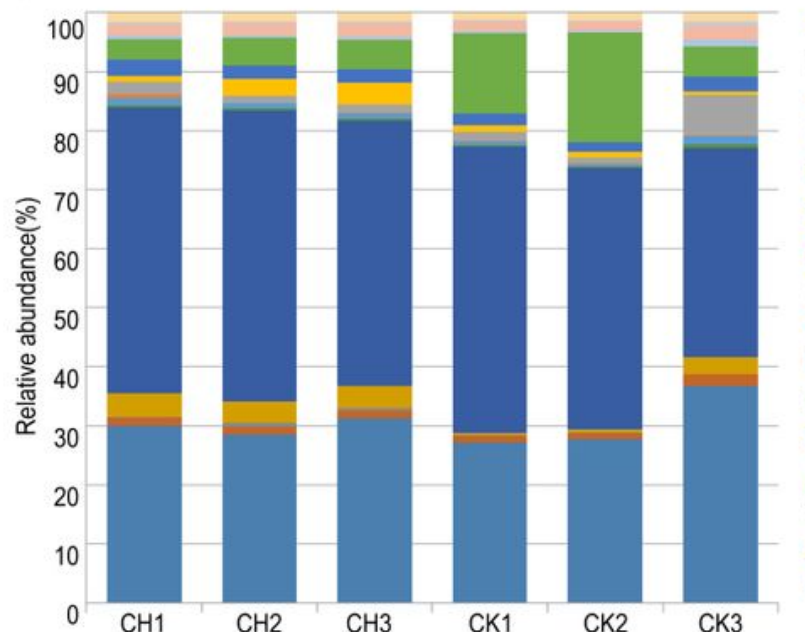

b

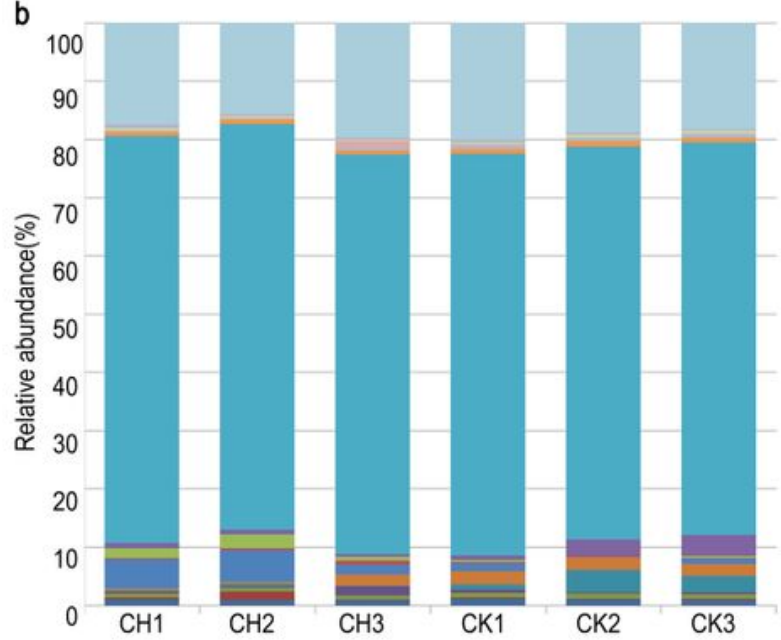

C

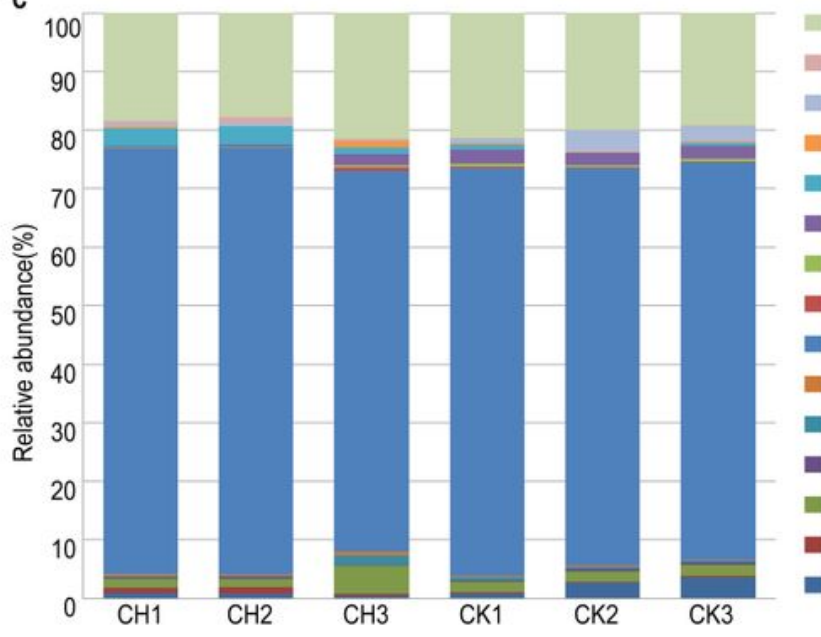

Others

Chloroflexi

Firmicutes

Planctomycetes

Thaumarchaeota

Actinobacteria

Nitrospirae

Acidobacteria

Ignavibacteriae

Bacteroidetes

Verrucomicrobia

Proteobacteria

Gemmatimonadetes

Crenarchaeota

Cyanobacteria

Unclassfied
= Others

- Streptomyces

Sulfuricella

Chloracidobacterium

Gemmatimonas

Bradyrhizobium

Unclassfied

Nitrospira

Nitrosopumilus

- Candidatus Koribacter

- Candidatus Nitrosoarchaeum

- Gemmatirosa

- Nitrososphaera

- Candidatus Solibacter

- Burkholderia

- Nitrosococcus

- Pseudomonas

\section{Others}

Candidatus Nitrosopumilus salaria

Nitrososphaera viennensis

Chloracidobacterium thermophilum

Candidatus Nitrosoarchaeum koreensis

Gemmatirosa kalamazoonesis

Gemmatimonas aurantiaca

uncultured Acidobacteria bacterium

Unclassfied

Candidatus Koribacter versatilis

Candidatus Solibacter usitatus

Sulfuricella denitrificans

uncultured bacterium

Candidatus Nitrosoarchaeum limnia

Nitrospira defluvii

\section{Figure 5}

Relative abundance of microbiota in the rhizosphere soil of chlorotic and normal plants. CH1-3. rhizosphere soil from chlorotic plants; CK1-3. rhizosphere soil from normal plants. Presented phylum (a), genus (b), and species (c) were defined as having a relative abundance greater than $0.5 \%$ in any one sample from chlorotic and normal plants. Relative abundance less than $0.5 \%$ in all samples at each taxonomic level were combined into the others. 


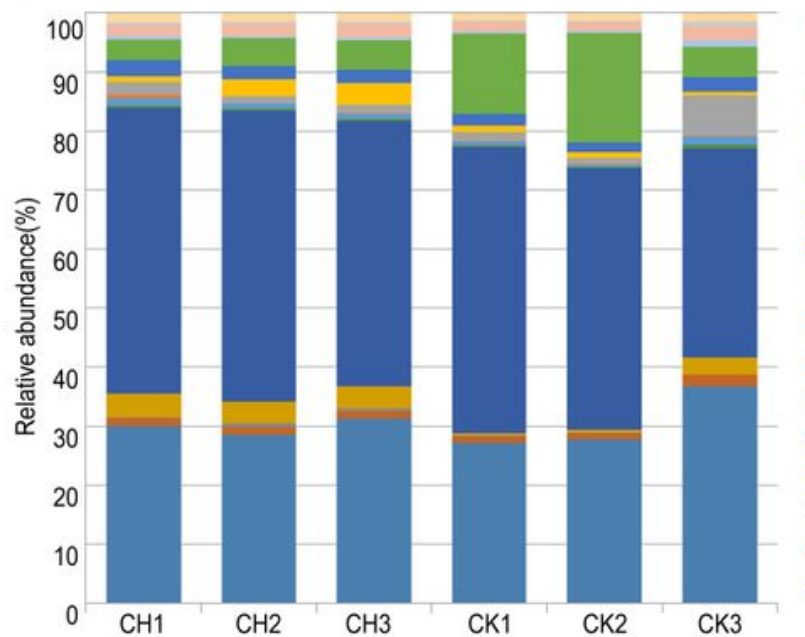

Others

Chloroflexi

Firmicutes

Planctomycetes

Thaumarchaeota

Actinobacteria

Nitrospirae

Acidobacteria

Ignavibacteriae

Bacteroidetes

Verrucomicrobia

Proteobacteria

Gemmatimonadetes

Crenarchaeota

Cyanobacteria

Unclassfied

b

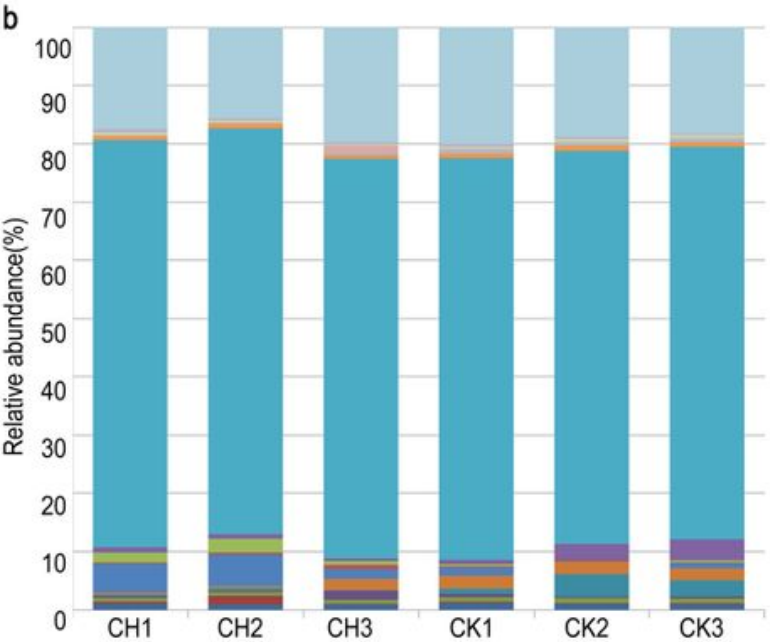

1- Others

- Streptomyces

- Sulfuricella

- Chloracidobacterium

- Gemmatimonas

- Bradyrhizobium

- Unclassfied

- Nitrospira

- Nitrosopumilus

- Candidatus Koribacter

- Candidatus Nitrosoarchaeum

- Gemmatirosa

- Nitrososphaera

- Candidatus Solibacter

- Burkholderia

- Nitrosococcus

- Pseudomonas

C

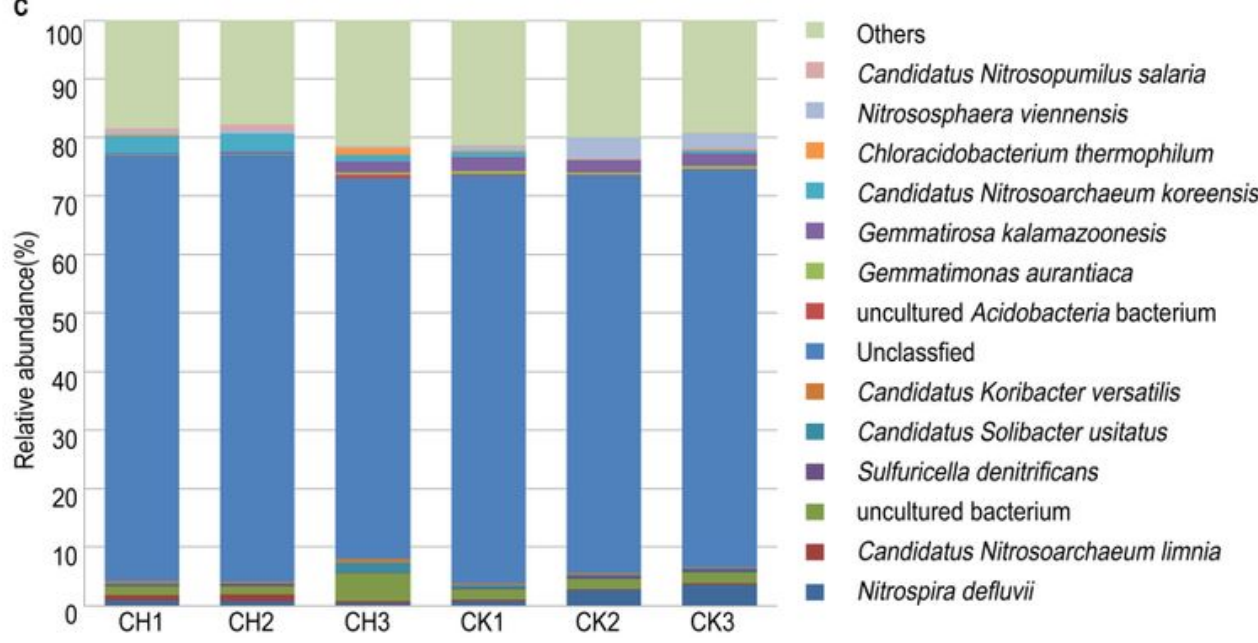

Figure 5

Relative abundance of microbiota in the rhizosphere soil of chlorotic and normal plants. $\mathrm{CH} 1-3$. rhizosphere soil from chlorotic plants; CK1-3. rhizosphere soil from normal plants. Presented phylum (a), genus (b), and species (c) were defined as having a relative abundance greater than $0.5 \%$ in any one sample from chlorotic and normal plants. Relative abundance less than $0.5 \%$ in all samples at each taxonomic level were combined into the others. 


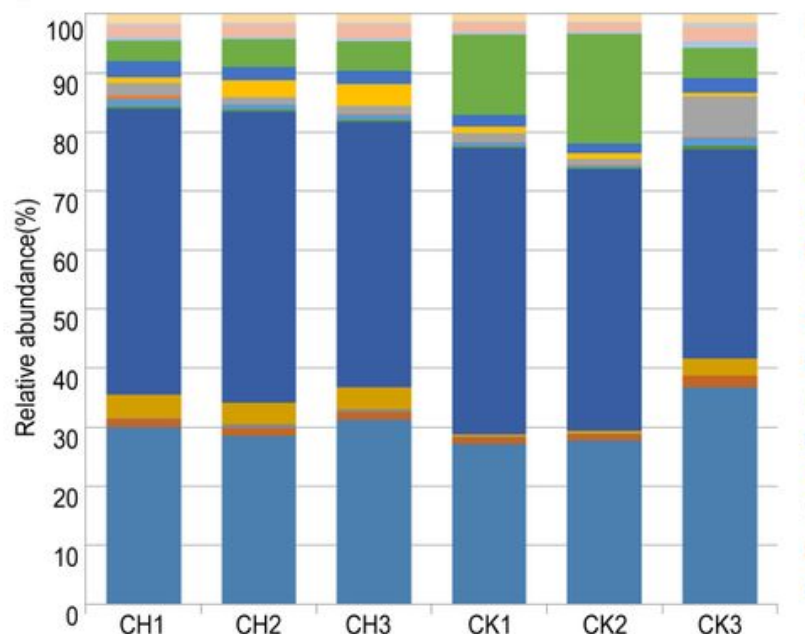

b

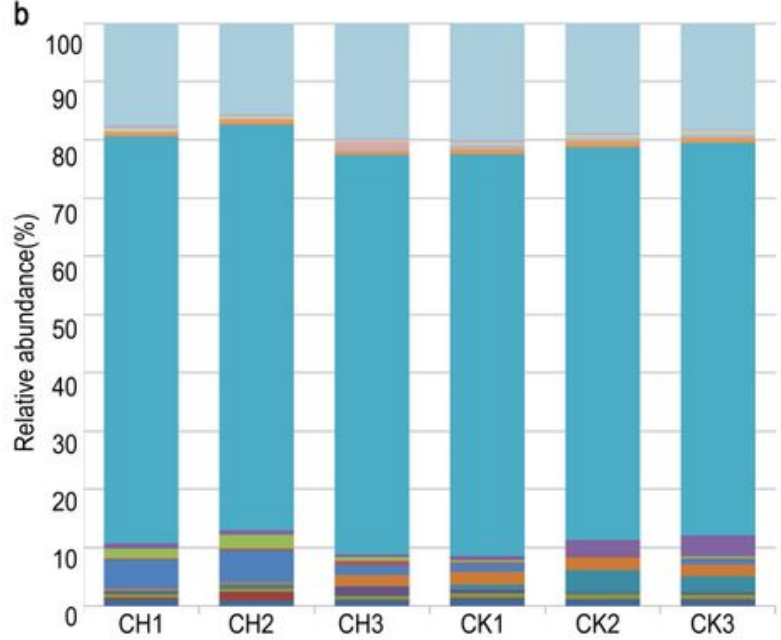

C

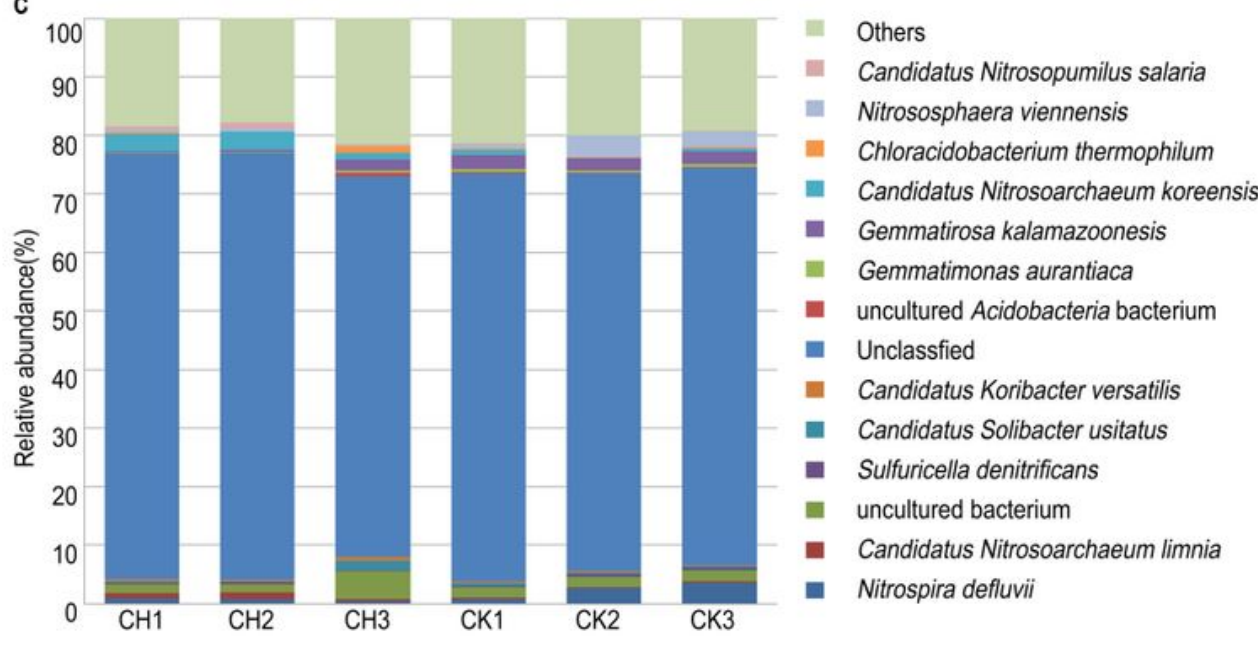

Others

Chloroflexi

Firmicutes

Planctomycetes

Thaumarchaeota

Actinobacteria

Nitrospirae

Acidobacteria

Ignavibacteriae

Bacteroidetes

Proteobacteria

Gemmatimonadetes

Crenarchaeota

Cyanobacteria

Unclassfied

= Others

- Streptomyces

Sulfuricella

Chloracidobacterium

- Gemmatimonas

Bradyrhizobium

Unclassfied

Nitrospira

Nitrosopumilus

- Candidatus Koribacter

- Candidatus Nitrosoarchaeum

- Gemmatirosa

- Nitrososphaera

- Candidatus Solibacter

- Burkholderia

- Nitrosococcus

- Pseudomonas

\section{Others}

Candidatus Nitrosopumilus salaria

Gemmatirosa kalamazoonesis

Gemmatimonas aurantiaca

uncultured Acidobacteria bacterium

Candidatus Solibacter usitatus

Sulfuricella denitrificans

uncultured bacterium
Verrucomicrobia

\section{Figure 5}

Relative abundance of microbiota in the rhizosphere soil of chlorotic and normal plants. CH1-3. rhizosphere soil from chlorotic plants; CK1-3. rhizosphere soil from normal plants. Presented phylum (a), genus (b), and species (c) were defined as having a relative abundance greater than $0.5 \%$ in any one sample from chlorotic and normal plants. Relative abundance less than $0.5 \%$ in all samples at each taxonomic level were combined into the others. 


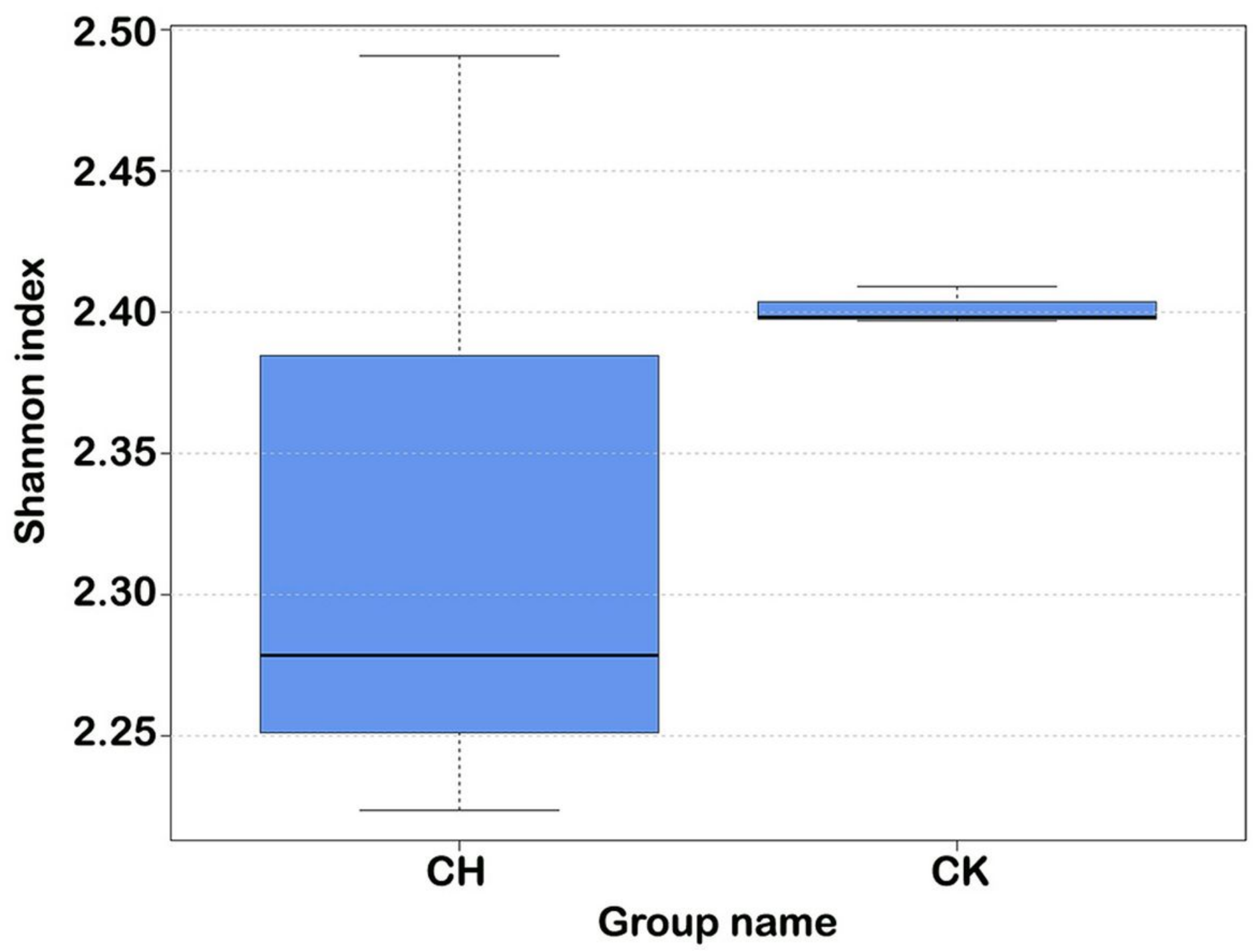

Figure 6

Shannon index of microbiome in the rhizosphere soil of chlorotic and normal plants. $\mathrm{CH}$. Rhizosphere soil of chlorotic plants, CK. Rhizosphere soil of normal plants. The larger the Shannon index, the better the sample uniformity. In the case of the same species richness, the greater the uniformity of each species in the community, the greater the diversity of the community 


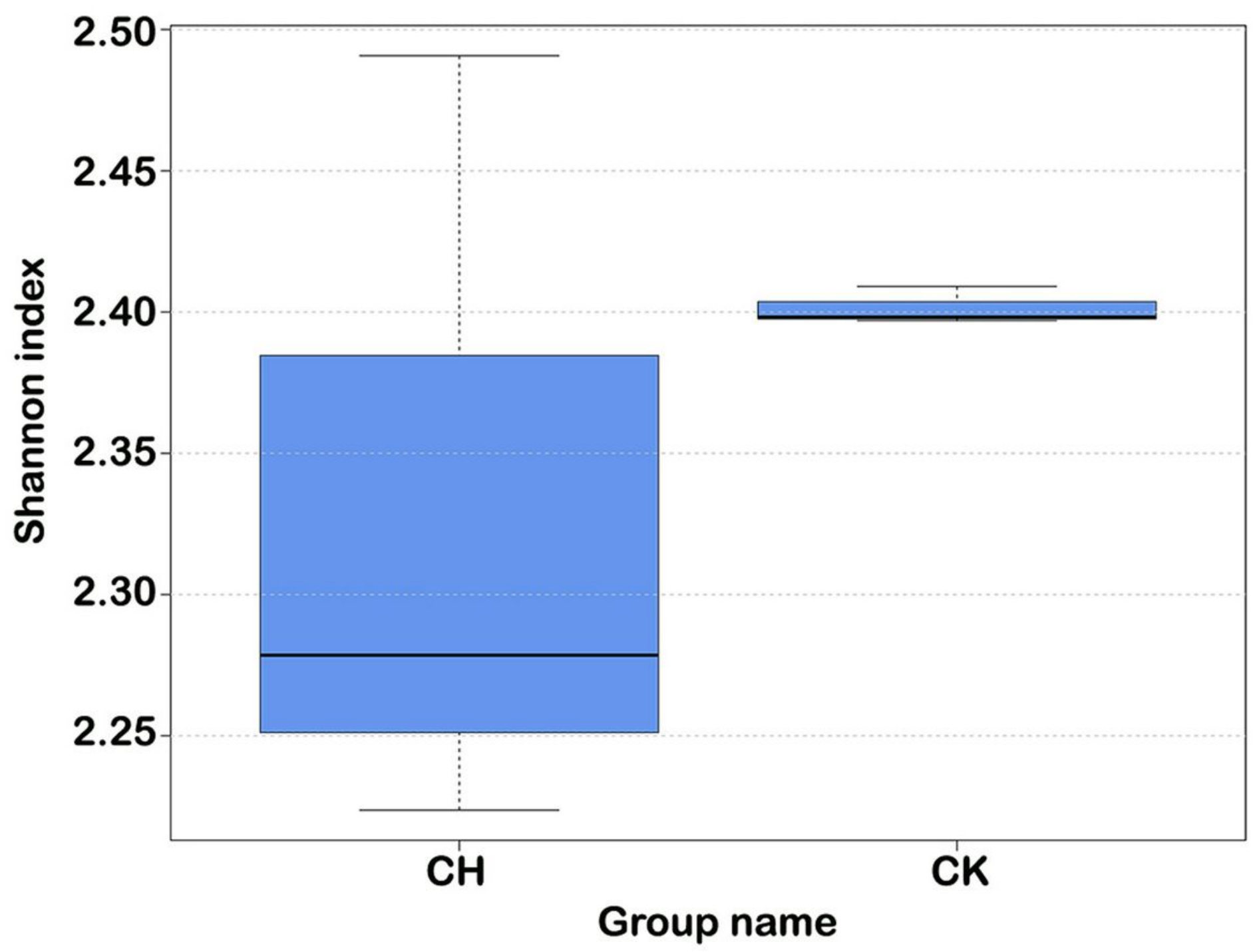

Figure 6

Shannon index of microbiome in the rhizosphere soil of chlorotic and normal plants. $\mathrm{CH}$. Rhizosphere soil of chlorotic plants, CK. Rhizosphere soil of normal plants. The larger the Shannon index, the better the sample uniformity. In the case of the same species richness, the greater the uniformity of each species in the community, the greater the diversity of the community 


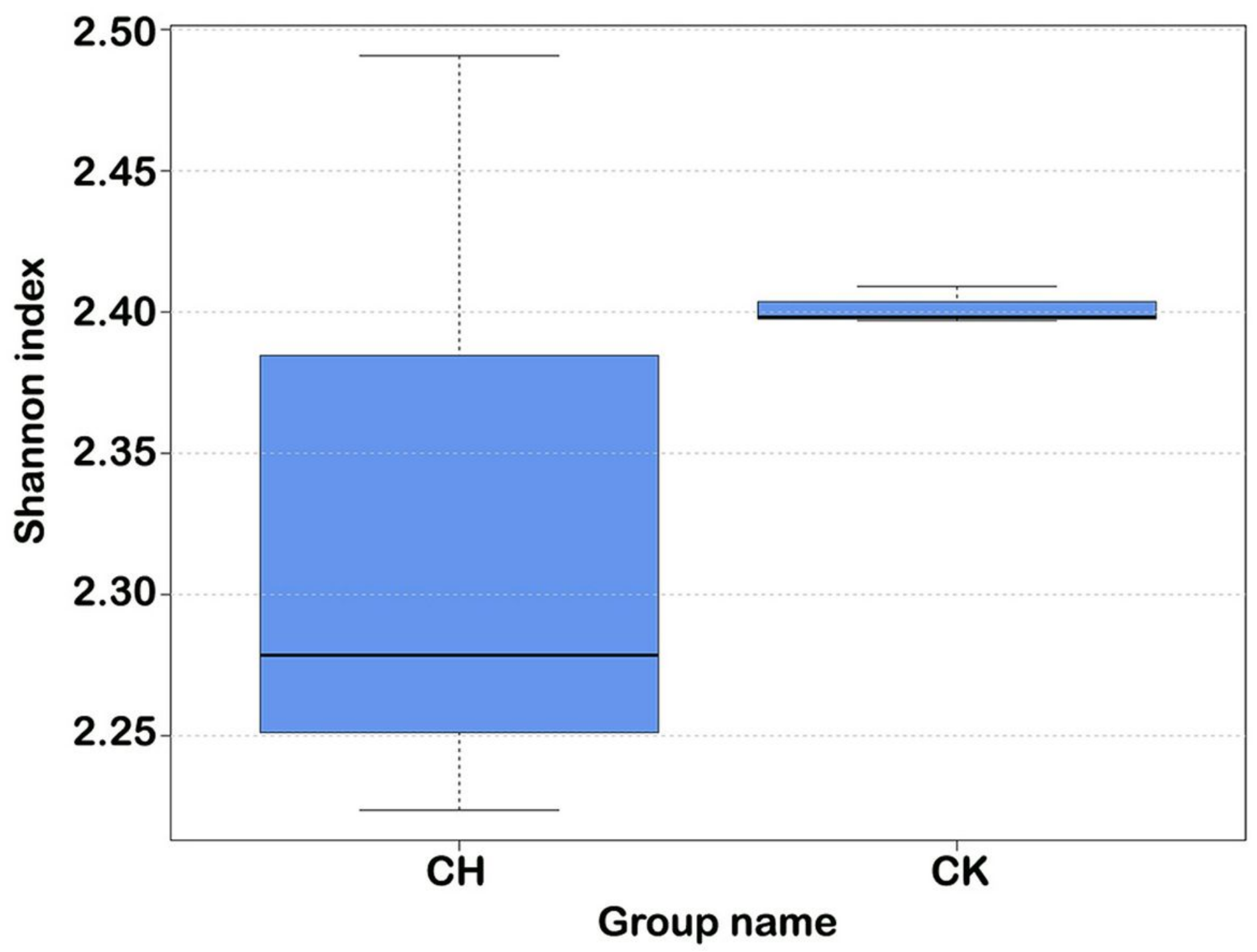

Figure 6

Shannon index of microbiome in the rhizosphere soil of chlorotic and normal plants. $\mathrm{CH}$. Rhizosphere soil of chlorotic plants, CK. Rhizosphere soil of normal plants. The larger the Shannon index, the better the sample uniformity. In the case of the same species richness, the greater the uniformity of each species in the community, the greater the diversity of the community 

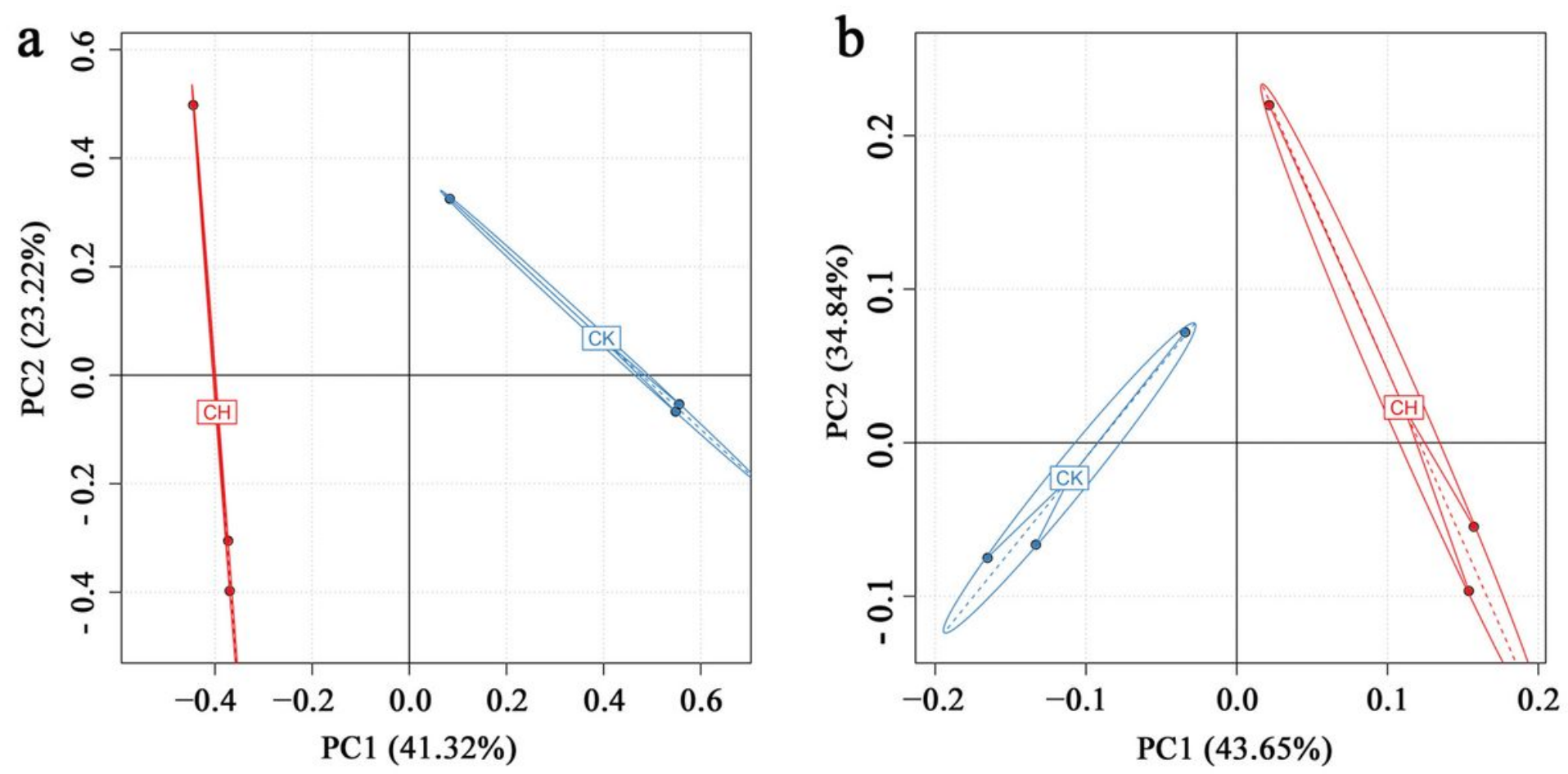

Figure 7

Principle component analysis based on the expressed genes annotated and abundance of bacterial species in the rhizosphere soil of chlorotic and normal plants. Each round-point represents a sample replicate, the abscissa is the value of principal component 1 , and the ordinate is the value of principal component 2. a. Expressed genes annotated; b. Abundance of bacterial species; $\mathrm{CH}$. Rhizosphere soil of chlorotic plants, CK. Rhizosphere soil of normal plants.
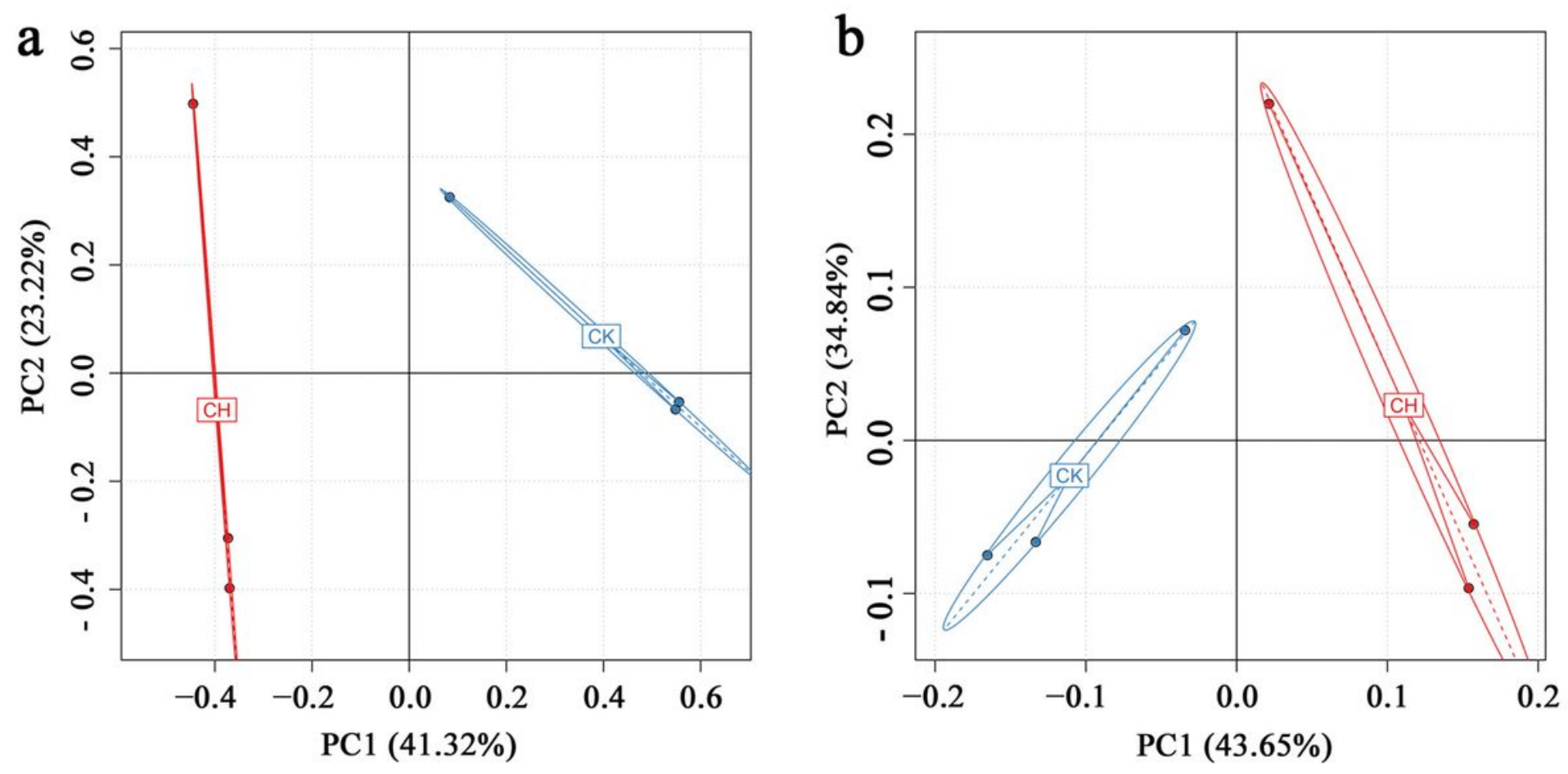

Figure 7 
Principle component analysis based on the expressed genes annotated and abundance of bacterial species in the rhizosphere soil of chlorotic and normal plants. Each round-point represents a sample replicate, the abscissa is the value of principal component 1 , and the ordinate is the value of principal component 2. a. Expressed genes annotated; $b$. Abundance of bacterial species; $\mathrm{CH}$. Rhizosphere soil of chlorotic plants, CK. Rhizosphere soil of normal plants.
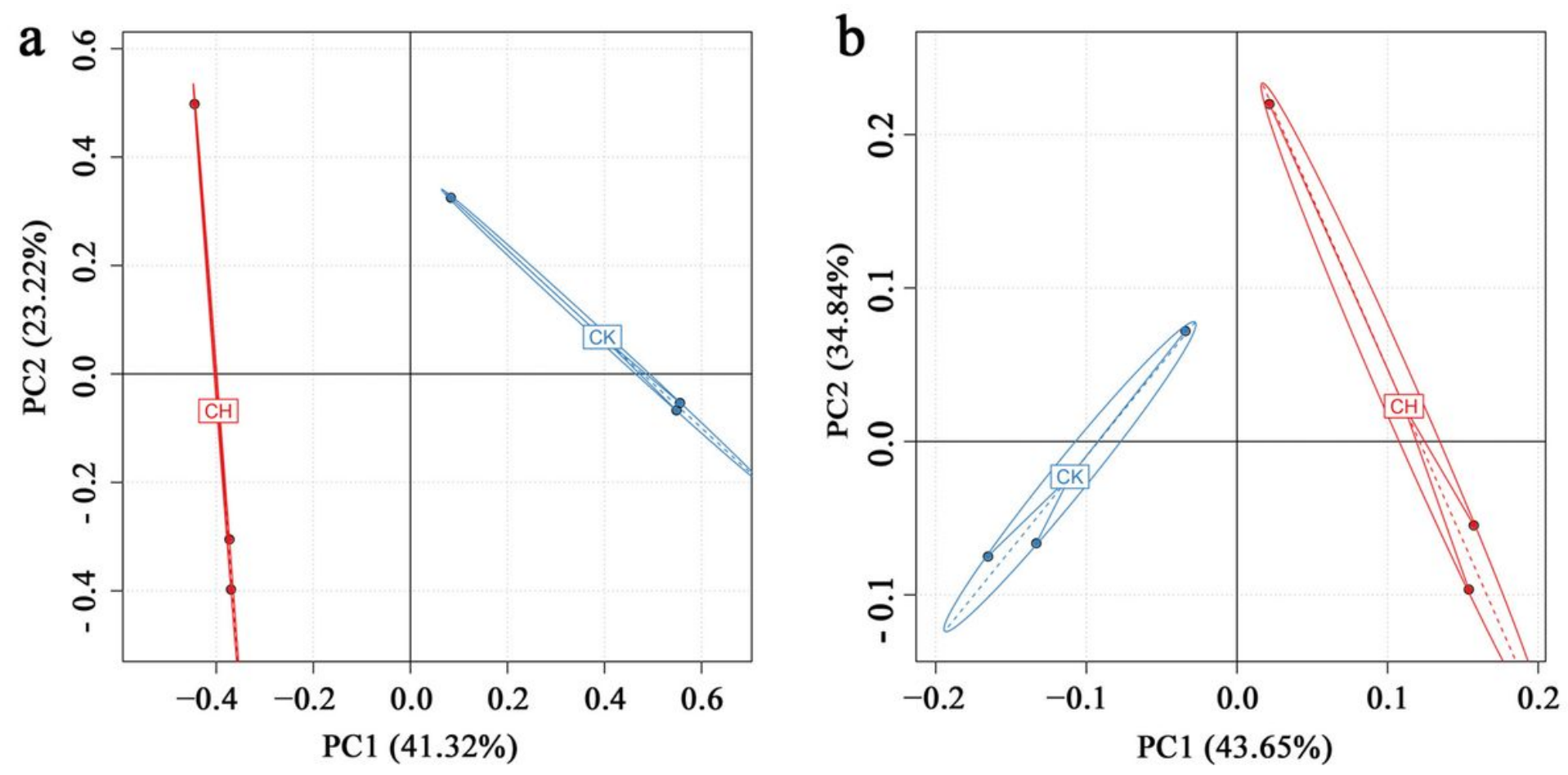

\section{Figure 7}

Principle component analysis based on the expressed genes annotated and abundance of bacterial species in the rhizosphere soil of chlorotic and normal plants. Each round-point represents a sample replicate, the abscissa is the value of principal component 1 , and the ordinate is the value of principal component 2. a. Expressed genes annotated; $b$. Abundance of bacterial species; $\mathrm{CH}$. Rhizosphere soil of chlorotic plants, CK. Rhizosphere soil of normal plants. 

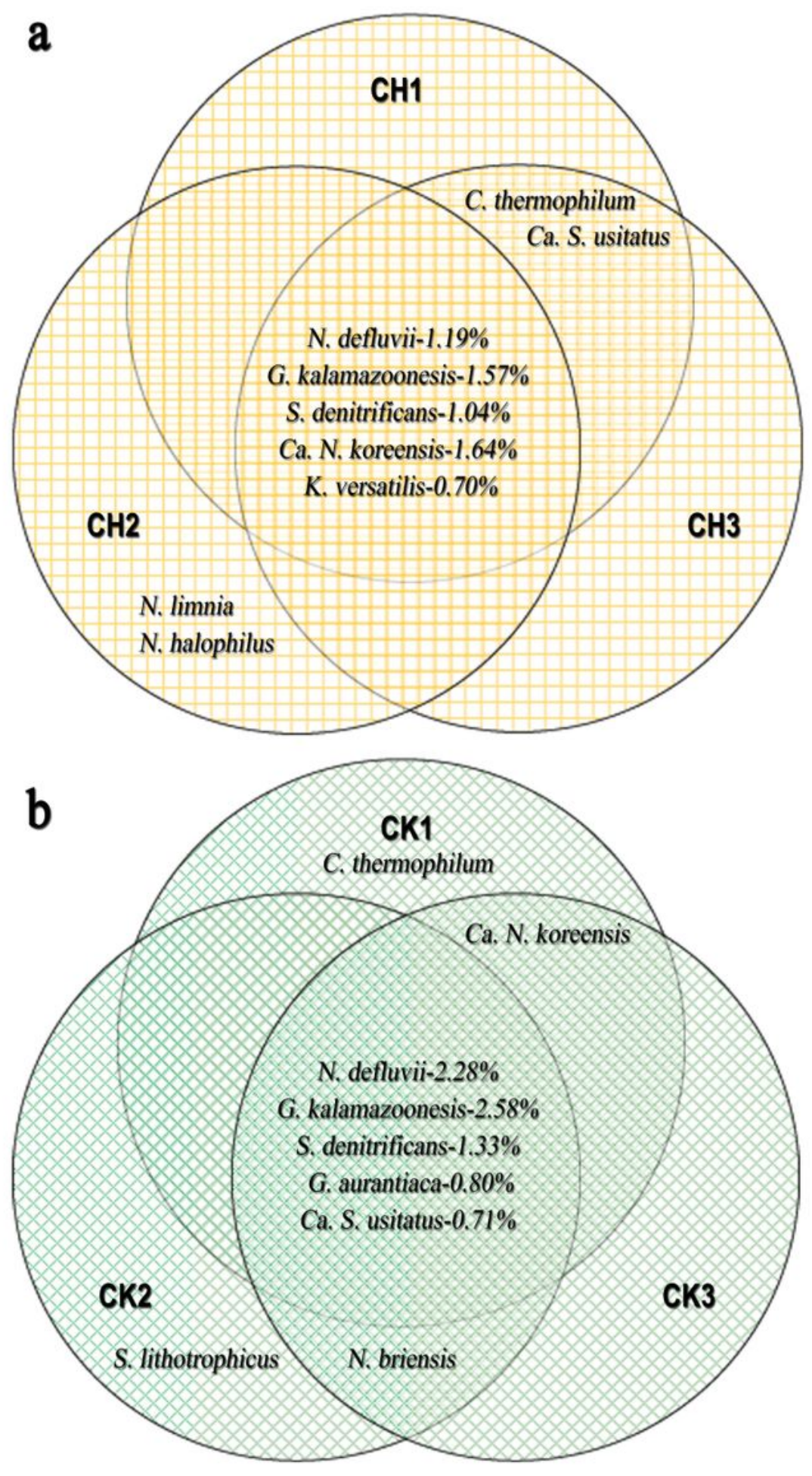

\section{Figure 8}

Simplified species composition in rhizosphere soil of chlorotic (a) and normal (b) plants. The five dominant species for $\mathrm{CH} 1$ were Nitrospira defluvii, Gemmatirosa kalamazoonesis, Sulfuricella denitrificans, Candidatus Nitrosoarchaeum koreensis, and Candidatus Koribacter versatilis. The five dominant species for CK1were Nitrospira defluvii, Gemmatirosa kalamazoonesis, Sulfuricella denitrificans, Gemmatimonas aurantiaca, and Candidatus Solibacter usitatus. 

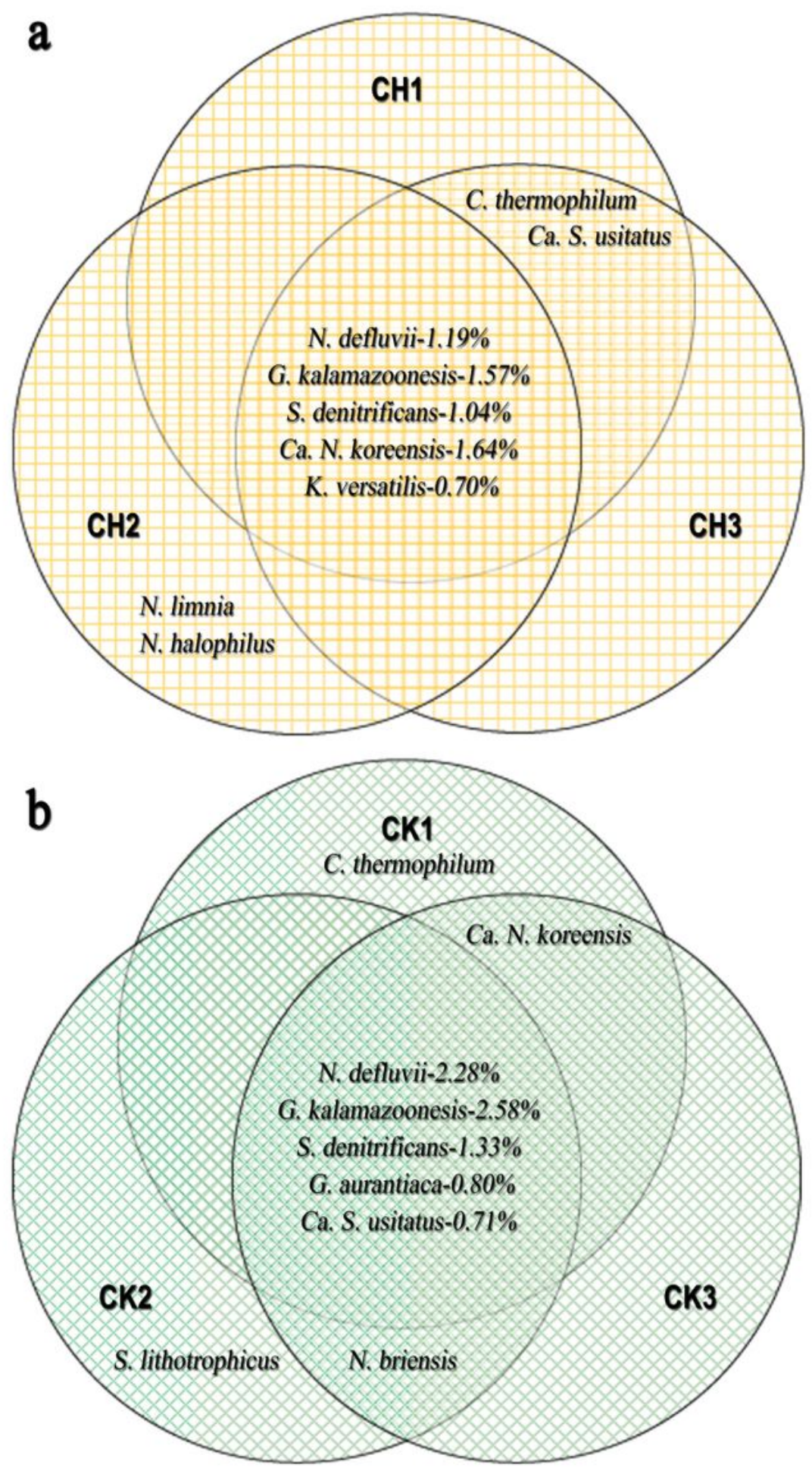

\section{Figure 8}

Simplified species composition in rhizosphere soil of chlorotic (a) and normal (b) plants. The five dominant species for $\mathrm{CH} 1$ were Nitrospira defluvii, Gemmatirosa kalamazoonesis, Sulfuricella denitrificans, Candidatus Nitrosoarchaeum koreensis, and Candidatus Koribacter versatilis. The five dominant species for CK1were Nitrospira defluvii, Gemmatirosa kalamazoonesis, Sulfuricella denitrificans, Gemmatimonas aurantiaca, and Candidatus Solibacter usitatus. 

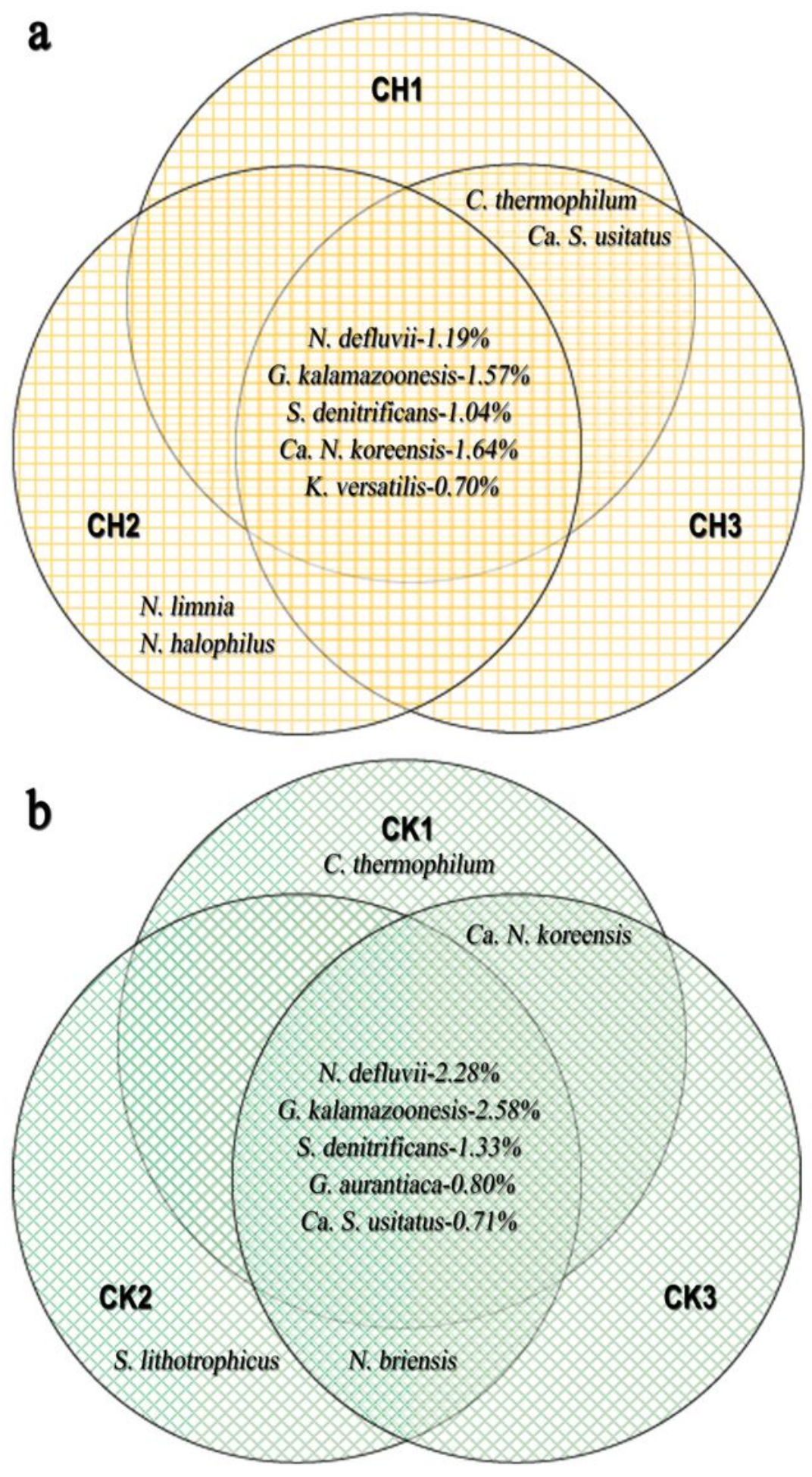

\section{Figure 8}

Simplified species composition in rhizosphere soil of chlorotic (a) and normal (b) plants. The five dominant species for $\mathrm{CH} 1$ were Nitrospira defluvii, Gemmatirosa kalamazoonesis, Sulfuricella denitrificans, Candidatus Nitrosoarchaeum koreensis, and Candidatus Koribacter versatilis. The five dominant species for CK1were Nitrospira defluvii, Gemmatirosa kalamazoonesis, Sulfuricella denitrificans, Gemmatimonas aurantiaca, and Candidatus Solibacter usitatus. 


\section{Supplementary Files}

This is a list of supplementary files associated with this preprint. Click to download.

- SupplementaryMaterial20201127.pdf

- SupplementaryMaterial20201127.pdf

- SupplementaryMaterial20201127.pdf 